\title{
Gene losses may contribute to subterranean adaptations in naked mole-rat and blind mole-rat
}

\author{
Zhi-Zhong Zheng ${ }^{\dagger, 1,2}$, Rong Hua ${ }^{\dagger, 1,2,3}$, Guo-Qiang Xu ${ }^{3,4}$, Hui Yang ${ }^{*}, 1$ Peng Shi* ${ }^{*}, 1,4,5,6$ \\ ${ }^{1}$ State Key Laboratory of Genetic Resources and Evolution, Kunming Institute of Zoology, Chinese Academy of Sciences, \\ Kunming 650223, China \\ ${ }^{2}$ Kunming College of Life Science, University of Chinese Academy of Sciences, Kunming 650204, China \\ ${ }^{3}$ College of Pharmaceutical Sciences, Soochow University, Suzhou 215006, China \\ ${ }^{4}$ Joint Laboratory of Animal Models for Human Diseases and Drug Development, Soochow University and Kunming Institute \\ of Zoology, Chinese Academy of Sciences, Kunming 650223, China \\ ${ }^{5}$ Center for Excellence in Animal Evolution and Genetics, Chinese Academy of Sciences, Kunming 650223, China \\ ${ }^{6}$ School of Future Technology, University of Chinese Academy of Sciences, Beijing 101408, China \\ ${ }^{\dagger}$ These authors contributed equally to this work.
}

*Correspondence to:

Peng Shi

Email: $\underline{\text { ship@,mail.kiz.ac.cn }}$

OR

Hui Yang

Email: yanghui@mail.kiz.ac.cn

State Key Laboratory of Genetic Resources and Evolution

Kunming Institute of Zoology, Chinese Academy of Sciences

32 Jiaochang Donglu, Kunming 650223, China

Phone: 86-871-68125411

Fax: 86-871-68125411

Key words: gene losses, naked mole-rat, blind mole-rat, subterranean adaptations 


\begin{abstract}
The naked mole-rats (Heterocephalus glaber, NMRs) and the blind mole-rats (Spalax galili, BMRs) are representative subterranean rodents that evolved many extraordinary traits, including hypoxia tolerance, longevity and cancer resistance. Although a batch of candidate loci responsible for these intriguing traits have been uncovered by genomic studies, many of them are limited to functional modifications of intact genes and little is known about the contributions of other genetic makeups. Here, to address this issue, we focused on gene losses (unitary pseudogenes) and systematically analyzed gene losses in NMRs and BMRs, as well as their respective terrestrial relatives, guinea pigs and rats, in a genome-wide scale. 167, 139, 341 and 112 pseudogenes were identified in NMRs, BMRs, guinea pigs and rats, respectively. Functional enrichment analysis identified 4 shared and 2 species-specific enriched functional groups (EFGs) in subterranean lineages. The pseudogenes in these EFGs might be associated with either regressive (e.g. visual system) or adaptive (e.g. altered DNA damage response) traits. In addition, several pseudogenes including TNNI3K and PDE5A, might be associated with their specific cardiac features observed in subterranean linages. Furthermore, we observed 20 convergent gene losses in NMRs and BMRs. Given that the functional investigations of these genes are generally scarce, we provided functional evidence that independent loss of TRIM17 in NMRs and BMRs might be beneficial for neuronal survival under hypoxia, supporting the positive role of eliminating TRIM17 function in hypoxia adaptation. We also demonstrated that pseudogenes, together with positively selected genes, reinforced subterranean adaptations cooperatively. Overall, our study provides new insights into the molecular underpinnings of subterranean adaptations and highlights the importance of gene losses in mammalian evolution.
\end{abstract}




\section{Introduction}

2 Subterranean niches provide mammalian dwellers with highly stressful living conditions that are characterized mainly by extreme hypoxia/hypercapnia, darkness, and food scarcity. Over the course of their evolution for millions of years, subterranean mammals have evolved exquisite physiological and morphological modifications to cope with these challenges (Nevo 1979). Excellent examples of subterranean adaptation are found in naked mole-rats (Heterocephalus glaber, NMRs) (Perez, et al. 2009; Seluanov, et al. 2009; Kim, et al. 2011; Smith, et al. 2011; Tian, et al. 2013; Park, et al. 2017; Zhao, et al. 2018) and the blind molerats (Spalax galili, BMRs) (Avivi, et al. 2001; Ashur-Fabian, et al. 2004; Shams, et al. 2004; Nasser, et al. 2005; Avivi, et al. 2010; Gorbunova, et al. 2012; Fang, Nevo, et al. 2014). NMRs and BMRs are phylogenetically distantly related subterranean rodents that evolved similar morphological and extraordinary physiological traits. To live in the burrow system, NMRs and BMRs evolved cylindrically shaped bodies, shortened limbs, strong claws, elongated incisors and degenerated visual system (Nevo 1979). They are highly tolerant to tissue hypoxia, resistant to oxidative stress induced by oxygen deficient and insensitive to acid induced by hypercapnia (Shams, et al. 2004; Kim, et al. 2011; Smith, et al. 2011; Fang, Nevo, et al. 2014; Park, et al. 2017). In addition, they are both extremely long-lived rodents that live more than 20 years and are able to suppress spontaneous and experimentally induced tumorigenesis (Seluanov, et al. 2009; Gorbunova, et al. 2012; Tian, et al. 2013), even though cases of cancer were reported recently in zoo-housed NMRs (Delaney, et al. 2016). They also evolved several species-specific traits, for example, eusociality (Jarvis 1981), poikilothermy (Buffenstein, et al. 2001) and lack of a circadian sleep rhythm in NMRs (Davis-Walton and Sherman 1994); enlarged brain structure related to sensing and orientation (Mann, et al. 1997; Kimchi and Terkel 2001), inconstant and changeful heart rate and increased muscle capillary density (Widmer, et al. 1997) in BMRs. These traits aroused general interests among researchers working on various filed such as evolutionary biology, genetics, aging and cancer research. Understanding the molecular bases of extraordinary traits in NMRs and BMRs may help us address some of the most challenging questions in biology and medicine, such as adaptations to extreme environments, mechanisms of hypoxia tolerance, mechanisms of aging and cancer resistance.

Genetic and genomic studies in NMRs and BMRs have uncovered the molecular bases of some extraordinary traits. For examples, genome sequencing revealed that the amino acid 
(AA) substitutions in NMR UCP1 protein are associated with its unique thermoregulation, and fast evolution of BMR embryonic haemoglobin gamma gene may contribute to hypoxia adaptation (Kim, et al. 2011; Fang, Nevo, et al. 2014). Comparative genomics has uncovered hundreds of positively selected genes (PSGs) and convergently evolved genes that may

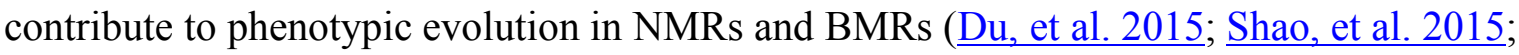
Davies, et al. 2018), including the convergence on the $S C N 9 A$ gene (encoding $\mathrm{Na}_{\mathrm{v}} 1.7$ ), which leads to the repeatedly evolved acid insensitivity (Fang, Nevo, et al. 2014; Liu, Wang, et al. 2014). However, it should be noted that the positive selection and convergence rarely occurred in the same gene across subterranean linages and that evolutionary changes in different genes might produce similar phenotypes (Davies, et al. 2018). In addition, transcriptome analysis identified many differentially expressed genes that are associated with longevity, hypoxia and hypercapnia tolerance (Kim, et al. 2011; Malik, et al. 2011; Malik, et al. 2012; Fang, Nevo, et al. 2014; Fang, Seim, et al. 2014). For example, two p53 target genes, CYTC and CASP9, are downregulated in BMRs under hypoxia, which may contribute to cellular protection against hypoxia induced apoptosis (Fang, Nevo, et al. 2014). Also, SMAD3 gene is upregulated in aging NMR brain, which may slow down the growth of cancer cells and contribute to cancer resistance (Kim, et al. 2011). Even though these studies identified many candidate loci that are potentially responsible for certain traits, functional assays are largely absent to validate their contributions. Moreover, most of these studies were focusing on the functional modifications of protein coding genes and many traits can still not be explained adequately. In this regard, to assess the contributions of other genetic makeups to subterranean adaptations is important and should not be ignored.

In addition to functional modifications of protein coding genes, other types of genetic changes can contribute to phenotypic evolution, such as pseudogenes (gene losses) (Albalat and Canestro 2016), regulatory element mutations (Wittkopp and Kalay 2012) and epigenetic modifications (Bonduriansky and Day 2009). Gene losses, which are usually associated with regressive evolution, were recently shown to play roles in adaptive phenotypic evolution more than we expected. Examples of adaptive gene losses include: i) in cetaceans, losses of DSG4, DSC1, TGM5, and GSDMA may contribute to the evolution of an epidermis morphology suit for aquatic environment and loss of POLM may contribute to the improved tolerance of oxidative DNA lesions (Sharma, et al. 2018; Huelsmann, et al. 2019); ii) in fruit bats, losses of FAMBB and FFAR3 may help them adapt to sugar-rich diet by increasing 
65 insulin secretion and insulin sensitivity (

66 PNLIPRP1 in herbivores may increase their capacity of fat storage, which is beneficial given

67 that herbivores usually consume fat-poor diet (Hecker, et al. 2019). These observations are in consistence with the "less is more" hypothesis (lson 1999). In terms of the subterranean mammals, however, almost all the studies addressing the role of gene losses in phenotypic evolution focused on eye degeneration (Kim, et al. 2011; Emerling and Springer 2014; Fang,

71 Nevo, et al. 2014; Fang, Seim, et al. 2014; Prudent, et al. 2016; Emerling 2018), while the contribution of gene losses to subterranean adaptations has yet to be investigated.

In this study, to assess the contribution of gene losses to subterranean adaptations, we systematically identified gene losses in NMRs and BMRs, as well as their terrestrial relatives, guinea pigs and rats, which were used as terrestrial controls. Comparison of gene losses between subterranean and terrestrial lineages identified a batch of pseudogenes that might be associated with specific subterranean physiological traits including an altered DNA damage response and a specialized cardiovascular system. In addition, we provided functional evidence for the contribution of eliminating TRIM17, a gene independently lost from NMRs and BMRs, to hypoxia adaptation. Thus, our work provides new insights into the molecular mechanisms of subterranean adaptations of both NMRs and BMRs. 


\section{Results and Discussion}

83

\section{Compositions of the pseudogene repertoires}

We first identified species-specific gene losses (unitary pseudogenes) in NMRs, BMRs, guinea pigs and rats by mapping a complete mouse protein set to their genomic sequences, followed by disablement (stop codons and frameshifts) detection and filtering by rigorous criteria (supplementary fig. S1; see Materials and Methods). The mouse genome was used as reference because mouse is currently the phylogenetically closest to NMRs and BMRs with high-quality genome assembly and annotations, which would mostly minimize the effects caused by phylogenetic distance. Although the difference in divergent time between each target species and mouse may affect the identified pseudogene numbers, the subsequent comparisons between subterranean and terrestrial control linages can help correct the phylogenetic bias as we focused on the proportion of functional similarity but not the absolute number of pseudogenes. Several factors affect the validity of detected gene losses including the misjudgement of orthologous sequences, genetic redundancy (Vavouri, et al. 2008; Qian, et al. 2010) and the false positives introduced by misjudgement of disruptive mutations, genome sequencing/assembly errors and annotation errors in reference genome. To address these issues and to obtain high-confidence gene losses for each species, we implemented a series of filtering steps (supplementary fig. S1; see Materials and Methods). Briefly, we first established the unitary status of each putative pseudogenic loci by i) removing loci hit by predicted proteins, intronless cDNA/expressed sequences or genes belong to large gene families such as olfactory receptors, zinc finger proteins and vomeronasal receptors; ii) removing genes with highly similar sequences at more than one locus in the genome; iii) removing genes without conserved genomic position between query species and mouse. Secondly, we removed false disruptive mutations generated by i) GeneWise alignment errors; ii) the sequencing and assembly errors; iii) potential annotation errors that resulted from the reference proteins with low transcript supporting level, or from downstream compensatory mutations that rescues the ORF disruption. As a result, 211, 211, 617 and 389 high-confidence unitary pseudogenes were identified from the NMRs, BMRs, guinea pigs and rats, respectively. To make our results more conservative, we further removed those pseudogenes without signatures of relaxed selection (supplementary fig. S1; see Materials and Methods) because gene dispensability and the following relaxed selective constraint is considered the hallmark of gene losses (Albalat and Canestro 2016). Finally, we 
114 obtained 167, 139, 341 and 112 gene losses in NMRs, BMRs, guinea pigs and rats,

115 respectively (fig. $\mathbf{1}$, table $\mathbf{1}$ and supplementary table $\mathbf{S 1}$ ).

117 Functional bias of gene losses in subterranean lineages

118 Next, we examined what kind of genes were preferentially lost in NMRs and BMRs, which

119 are more likely to be associated with subterranean adaptations. Using mouse orthologs as

120 surrogates, we firstly obtained all associated GO terms for all pseudogenes in each species.

121 Functionally cross-related GO terms were clustered into functional groups and a subset of

122 GO terms from each group that are overrepresented in subterranean lineage pseudogenes

123 compared to subterranean lineage non-pseudogenes or control lineage pseudogenes (NMRs

$124 v s$. guinea pigs and BMRs vs. rats) were identified as an enriched functional group (EFGs)

125 (see Materials and Methods). Among the 24 clustered functional groups, a total of 10 EFGs

126 were identified, with 5 in the NMR pseudogenes and 5 in the BMR pseudogenes (fig. 2 and

127 supplementary table S2). For each EFG, the proportion of pseudogenes from the

128 subterranean lineage is significantly higher than the pseudogenes from the corresponding

129 control lineage and/or the functional genes from their own genome (fig. 2 and

130 supplementary table S2). Genes in two EFGs might be associated with species-specific

131 traits. For example, the EFG for "lipid metabolism" might be associated with the alternating

132 membrane phospholipid composition in NMRs (Riccio and Goldman 2000; Mitchell, et al.

133 2007). The EFG "neural processes" might be associated with the enlarged brain volume in

134 BMRs (Mann, et al. 1997; Kimchi and Terkel 2001). Four EFGs, namely, those for the

135 "visual system", "reproduction", "DNA damage response" and "proteolysis", were shared

136 between the pseudogene lists from the two subterranean species. These repeatedly observed

137 EFGs support a notion that subterranean adaptations are relatively restricted to where certain

138 physiological process was recurrently modified to cope with stressful environments.

139 The EFG for "DNA damage response" is a good example to illustrate this notion. The

140 highly hypoxic and hypercapnic burrow environments cause a significant oxidative stress on

141 both BMRs and NMRs (Andziak, et al. 2006; Caballero, et al. 2006). The oxidative stress

142 under hypoxic environment can cause DNA damages, especially DNA double-stranded

143 breaks (DSBs) (Barzilai and Yamamoto 2004). We observed significant enrichments of NMR

144 and BMR pseudogenes in "DNA damage response" EFG when compared to their own

145 functional gene background (FDR corrected $P=0.0081$ for NMRs and $P=0.0024$ for BMRs, 
146 Fisher's exact test) (supplementary table S2), while guinea pig and rat pseudogenes show

147 no significance compared to their respective functional gene background (supplementary

148 table S2). The genes in this EFG involve DNA damage checkpoint and DNA repair

149 regulation, including D7ERTD443E, NUDT16L1 and EME2 in NMRs and NEK11,

150 D330045A20RIK and EME2 in BMRs (fig 3A and supplementary table S2 and S3).

151 Among them, D7ERTD443E (FATS) and NEK11 are checkpoint related genes that affect cell

152 cycles and apoptosis by regulating p53/p21 and CDC25A, respectively (Melixetian, et al.

153 2009; Li, et al. 2010; Zhang, Zhang, et al. 2010; Yan, et al. 2014) (fig. 3A). Interestingly,

154 both FATS and NEK11 are frequently deleted or downregulated in various human tumours

155 (Zhang, Zhang, et al. 2010; Liu, Gao, et al. 2014), reflecting a beneficial effect of their

156 functional losses on tumour cell survival, which is essential for tumor progression. This

157 beneficial effect may probably be utilized by NMRs and BMRs to cope with hypoxic stress,

158 particularly, prevent hypoxic cell death. The roles of FATS and NEK11 in tumour

159 progression and hypoxia adaptation can be reconstituted by a Spalax p53 mutation, which

160 mimics a human oncogenic mutation of p53 Lys-174 and was thought to be adaptive to

161 hypoxia (Ashur-Fabian, et al. 2004). Supporting this idea, the NMR fibroblasts were shown

162 to have a lower $\mathrm{p} 21$ response to $\gamma$-irradiation (IR), a reduced sensitivity to IR-induced

163 senescence and resistance to IR-induced apoptosis (Zhao, et al. 2018).

164 DNA repair is another critical theme in DNA damage response and both NMRs and BMRs

165 have evolved a higher DNA repair efficiency (Petruseva, et al. 2017; Domankevich, et al.

166 2018). Two negative DNA repair regulators, NUDT16L1(TIRR) and D330045A20RIK

$167(R A D X)$, were detected as pseudogenes in NMRs and BMRs, respectively (supplementary

168 table S2 and S3). TIRR is a Tudor interacting repair regulator that inhibits the activity of

169 53BP1 and this inhibitory effect is utilized by cancer cells to increase genomic instability as

170 TIRR locus was observed to be amplified in human cancers (Drane, et al. 2017). Depletion or

171 silencing of TIRR markedly reduces the nuclear soluble levels of 53BP1, resulting in

172 enhanced phosphorylation and association of 53BP1 with its effector proteins after induction

173 of DNA damage (Drane, et al. 2017). In this regard, TIRR loss might help NMRs improve the

174 DSBs repair efficiency by releasing more 53BP1 protein and enhancing 53BP1 activity in

175 DNA repair. $R A D X$ encodes a single-strand DNA binding protein that prevents fork collapse

176 by modulating RAD51 accumulation on replication forks (Dungrawala, et al. 2017). RAD51

177 is critical to reversal fork formation, reversed forks protection and homologous 
recombination repair (HR). However, either too much or too little of RAD51 accumulation on forks can cause DNA damage and fork collapse. RADX, acting like a buffer, competes with RAD51 for binding to single-stranded DNA and ensures the right amount of RAD51 for reversal fork formation and protection (Bhat, et al. 2018). In fact, in normal cells, RADX inactivation leads to excessive RAD51 activity and DNA damages, despite RAD51 functioning as a DSB repair protein. In contrast, RADX inactivation in $B R C A 2$-deficient cancer cells, in which RAD51 function is reduced, is sufficient to rebalance RAD51 activity, protect forks from MRE11- and DNA2-dependent fork degradation (Dungrawala, et al. 2017; Bhat, et al. 2018). Due to the hypoxic environments, subterranean mammals suffer oxidative stress frequently, which causes replication stress and DNA lesions (Domankevich, et al. 2018) and prefer a higher RAD51 activity like BRCA2-deficient cells. Therefore, RADX loss in BMRs might contribute to the enhanced DNA repair efficiency (Domankevich, et al. 2018) by rebalancing RAD51 functions, protecting against replication fork degradation and enhancing HR under a stressful condition. Interestingly, EME2, an endonuclease component gene that is capable of causing DNA damage under replication stress (Amangyeld, et al. 2014; Pepe and West 2014; Techer, et al. 2016), was independently lost from NMRs and BMRs (fig. 3A and supplementary table S2 and S3), which might help maintaining genome integrity by removing the potential source of DNA damages. Such observations suggest a contribution of these pseudogenes to the enhanced DNA repair efficiency in both species. Taken together, the similar direction of evolution of DNA damage response, i.e., attenuating apoptosis and enhancing DNA repair efficiency, indicates a functional convergence between NMRs and BMRs and highlights the importance of maintaining genome integrity under hypoxic conditions.

In addition to the shared EFGs, the pseudogenes in a functional group named “cardiovascular system" also show a functional convergence, even though no significant enrichments were observed (fig. 3B and supplementary table S2). This convergence might be associated with the low blood pressure in NMRs and low heart rate in BMRs, and both of them seem to be caused by changes in cardiac contraction (Arieli and Ar 1981; Widmer, et al. 1997; Grimes, Reddy, et al. 2014; Grimes, Voorhees, et al. 2014). In NMRs, TNNI3K and RNPEP were detected as pseudogenes (supplementary table $\mathbf{S 3}$ ). A recent study also reported that $T N N I 3 K$ was pseudogenized in NMRs (Gan, et al. 2019). These two genes are involved in the contraction and action potential repolarization of cardiac muscle and blood 
210 pressure regulation. TNNI3K is a functional kinase and was shown to directly interact with

211 cardiac troponin I ( Zhao, et al. 2003). The overexpression of TNNI3K or its knockdown was

212 shown to enhance or decrease the contraction of cardiomyocytes, respectively (Wang, Wang,

213 Song, et al. 2013; Wang, Wang, Su, et al. 2013). In line with this, the natural knockout of

214 TNNI3K in NMRs coincides with its low resting cardiac contractility (Grimes, Voorhees, et

215 al. 2014) (fig. 3B). The other pseudogene, RNPEP, encodes Aminopeptidase B (Aurich-

216 Costa, et al. 1997). Inhibition of RNPEP was shown to suppress the development of

217 hypertension in spontaneously hypertensive rats (Aoyagi, et al. 1986), supporting its role in

218 blood pressure regulation. Therefore, the abnormal regulation of blood pressure by losing

$219 R N P E P$, together with low cardiac contractility by losing TNNI3K, might contribute to low

220 blood pressure observed in NMRs (Grimes, Reddy, et al. 2014) (fig. 3B). In BMRs, PDE5A,

221 which encodes a wide studied cGMP esterase, was detected as a pseudogene (supplementary

222 table S3). PDE5A is involved in various cardiomyopathies including cardiac hypertrophy

223 and myocardial infarction (Takimoto, et al. 2005; Perez, et al. 2007; Tedford, et al. 2008;

224 Giannetta, et al. 2012). Interestingly, like TNNI3K, the inhibition of PDE5A was also shown

225 to associate with suppressed contractility (Borlaug, et al. 2005) (fig. 3B). Even though the

226 cardiomyocyte contractility in BMRs has yet to be determined, the heart rate of BMRs was

227 observed to be lower than rat under both normoxia and hypoxia conditions (Arieli and Ar

228 1981), which might be caused by lowered cardiac contractility. Interestingly, inhibition of

$229 T N N I 3 K$ or PDE5A was shown to be cardioprotective against several cardiomyopathies

230 (Takimoto, et al. 2005; Perez, et al. 2007; Tedford, et al. 2008; Giannetta, et al. 2012;

231 Vagnozzi, et al. 2013). Moreover, TNNI3K inhibition was shown to increase the frequency of

232 the mononuclear diploid cardiomyocyte (MNDCM) population and to elevate cardiomyocyte

233 proliferation after injury (Patterson, et al. 2017; Gan, et al. 2019) (fig. 3B). In this regard, the

234 cardioprotective effects conferred by $T N N I 3 K$ or PDE5A losses in each species are likely to

235 be adaptive because cardiomyocytes are vulnerable to oxidative stress, which poses a

236 challenge to cardiomyocyte survival (Gottlieb, et al. 1994; Yellon and Hausenloy 2007).

237

238 Potential roles of convergent gene losses (CGLs) in subterranean adaptations

239 It has been shown recently that CGLs contribute to repeatedly evolved adaptations (Meyer, et

240 al. 2018; Sharma, et al. 2018; Hecker, et al. 2019). To reveal subterranean adaptations that

241 were contributed by CGLs, we compared the pseudogene lists from NMRs and BMRs. 20 
CGLs were observed between NMRs and BMRs (supplementary table S3 and S4). The hypergeometric test shows that these CGLs significantly outnumbered the random expectation $\left(1.42, P=1.25 \times 10^{-17}\right.$, fig. 4B). To examine the extent to which the number of CGLs are affected by similarity of ecological niches, we further paired the four species according to the similarities and differences in their living environments. Among 4 pairs of species, NMRs-BMRs (N-B) and guinea pigs-rats (G-R) live in similar environment (homotypic pair), while NMRs-rats (N-R) and BMRs-guinea pigs (B-G) live in different environment (heterotypic pair). Comparison of pseudogene lists between paired species shows that homotypic N-B pair is the most prominent with highest number of CGLs (fig. 4A). In contrast, the number of CGLs from homotypic G-R pair is significantly lower than NB pair (5/453 vs. 20/306, $P<0.0001$, Fisher's exact test) (fig. 4A). The CGLs number from the homotypic N-B pair is significantly higher than that from heterotypic B-G pair (20/306 vs. $7 / 480, P=0.0002$ ), while there is no significant difference between the CGLs number from the homotypic G-R pair and that from the heterotypic N-R pair (fig. 4A). These results suggest that the CGLs between NMRs and BMRs might result from the similar response to lifestyle transition from aboveground to underground and probably contribute to the evolution of their shared traits.

To associate overlapping pseudogenes with subterranean traits, we leveraged knockout mice phenotypic information (MPI) from literatures or MGI database and genetic associations from the public NHGRI-EBI GWAS catalog (MacArthur, et al. 2017). Degenerated visual capacity is one of the most significant traits that shared between NMRs and BMRs. By examining the MPI, we found that 5 convergent gene losses, including GUCY2F, ABCB5, RP1L1, CRB1 and ARR3 (Deming, et al. 2015), are vision related and knockout mice of each gene show visual abnormality (supplementary table S5). It is worth noting that we also identified many other vision related genes that lost specifically in each species, including genes reported by previously studies (Kim, et al. 2011; Emerling and Springer 2014; Fang, Nevo, et al. 2014; Prudent, et al. 2016; Emerling 2018; $\underline{\text { Sharma, et al. }}$ 2018), and new vision related pseudogenes, such as CRB1 in BMRs (supplementary table S6). Thus, these gene losses collectively contribute to the eye degeneration in NMRs and BMRs. We also searched the significant genetic associations $(P<1 \mathrm{e}-06)$ for all overlapping genes that curated in the NHGRI-EBI GWAS catalog. Interestingly, we found that variants in 5 genes, including $A B C B 5, L E T M 2, I N M T, R P 1 L 1$ and $C R B 1$, are significantly associated 
with cardiovascular traits including PR interval, pulse pressure and systolic blood pressure (Deng, et al. 2013; Evangelou, et al. 2018; Feitosa, et al. 2018; Ramírez, et al. 2018; Giri, et al. 2019)( supplementary table S7). Among them, except that SNP in INMT gene is located in coding region, all of the rest variants are located in regulatory regions or introns. Even though further investigations are needed, these data imply the contributions of losing such genes to cardiovascular adaptations in both NMRs and BMRs, especially blood pressure control under hypoxia. More importantly, we noticed that $A B C B 5, R P 1 L 1$ and $C R B 1$ are also vision related pseudogenes. Therefore, these genes may control multiple traits, in this case, the degenerated eyes and cardiovascular traits, simultaneously. This phenomenon is also observed in other vision related genes. For example, knockout mice of BFSP1, a BMRs vision related pseudogene, show both visual abnormalities and enlarged heart (MP:0000274). The phenotypic effects outside the visual system are indicative of gene pleiotropy and arise a question of why NMRs and BMRs lost these genes despite their non-visual functions. One possibility is that the non-visual phenotypic effects generated by losing such visual genes provides survival benefits because they affect cardiac features, which is important for hypoxia adaptation. If this is the case, some vision related gene losses should not simply be interpreted as the consequences of relaxed selection, rather, they probably have played positive roles in subterranean adaptations. A recent study in human populations demonstrated that many myopia-related mutant alleles are associated with reproductive benefits, and the reproduction-related selection probably contributes to the myopia epidemic inadvertently (Long and Zhang 2020). This is well supporting our above hypothesis. When further investigations are conducted and more data are accumulated in the future, we expect to have a better understanding of this issue.

The functional studies also provide clues regarding the potential roles of gene losses in subterranean adaptations. For example, as mentioned earlier, loss of EME2 could probably alleviate DNA damages induced by oxidative stress under hypoxic condition (Amangyeld, et

300 al. 2014; Pepe and West 2014; Techer, et al. 2016). Another identified pseudogene, TRIM17, 301 is also quite intriguing. It has been suggested that TRIM17 is involved in trophic factor 302 withdrawal-induced neuronal apoptosis (Lassot, et al. 2010). Considering that both NMRs 303 and BMRs are highly tolerant to hypoxia induced brain injury (Avivi, et al. 2010; $\underline{\text { Park, et al. }}$ 304 2017), it is possible that independent loss of TRIM17 in NMRs and BMRs contribute to protecting neural cells against hypoxia induced apoptosis. However, no direct links have been 
established between hypoxic stress and the actions of TRIM17, neither in vivo nor in vitro. It is also possible that other uncharacterized functions of TRIM17 are associated with some other subterranean traits. For example, TRIM17 knockout mice show physiological traits that are not involved in nerve system, including increased circulating alkaline phosphatase level (MP: 0002968) and increased circulating thyroxine level (MP: 0005477) (supplementary table S5). Therefore, functional investigations for these less well-characterized genes would provide insights into the roles of their functional losses in subterranean adaptations.

314 Functional loss of TRIM17 contributes to neuroprotection under hypoxia in vitro

315 We then chose TRIM17 to perform the functional survey to check whether loss of TRIM17 is

316 involved in protecting neural cells against hypoxia-induced apoptosis, as preventing cell

317 death and injury, especially neuronal cells, is a critical theme in adapting to hypoxic

318 environments (Ramirez, et al. 2007). First, we examined the pseudogenization status of

319 TRIM17 and their possible correlation with hypoxia. Disruptive mutations were confirmed by 320 manual inspection of the alignments, showing that TRIM17 is inactivated by one premature stop codon in NMRs and 1 stop codon/4 indels in BMRs (fig. 5A). The RING finger domain of TRIM17 is used to execute its E3 ligase function (Lassot, et al. 2010), however, it is disrupted in both species. We also examined the frequency of disruptive mutations in 11 BMR populations ( $\mathrm{Li}$, et al. 2015), and no segregations were found (Data not shown). In NMRs, the premature stop codon was also verified by the available transcriptomic reads and genomic sequence of another individual (supplementary table S3). Furthermore, TRIMI7 shows signature of relaxed selective constraint in both species, even though not significant (NMRs: $k=0.69, P=0.1338$; BMRs: $k=0.72, P=0.1214$ ) (supplementary table $\mathbf{S 1}$ ). These results together confirmed the elimination of an intact TRIM17 in both NMRs and BMRs. Interestingly, we noticed that TRIM17 was independently lost at least two times in cetaceans, with a shared $1 \mathrm{bp}$ deletion in toothed whales (Monodontidae, Phocoenidae and Delphinidae) (Sharma, et al. 2018) (fig.5B) and a shared premature stop codon in baleen whales (Balaenopteridae) (fig.5C). Inactivating mutations were also detected in several species in other families including right whale (Eubalaena japonica), pygmy sperm whale(Kogia breviceps) and boutu (Inia geoffrensis) (Data not shown). Moreover, TRIM17 was also lost in two pangolins (Manis pentadactyla and Manis javanica) due to a shared premature stop codon (fig. 5D) (Sharma, et al. 2018). Like NMRs and BMRs, cetacean and 
pangolins experience hypoxic stress during their lifetime (Weber, et al. 1986; $\underline{\text { Ramirez, et al. }}$ 2007; Choo, et al. 2016). Therefore, independently losses of TRIMI7 in distinct hypoxia tolerant taxa probably suggest a relevance of TRIM17 losses in hypoxia adaptation.

To illustrate this idea, we next examined the changes in Trim17 expression in response to 342 hypoxia in both Neuro-2a (N2a) cells and mouse primary cortical neurons. An immunoblot analysis shows that in contrast to trophic factor withdrawal, the Trim 17 protein level is significantly decreased in both N2a cells and primary neurons after treating with hypoxic stress $\left(1 \% \mathrm{O}_{2}\right)$ (fig. 5E-F), suggesting a hypoxia-responsive regulatory mechanism for Trim17 protein. We further tested whether Trim17 knock-down confers a neuroprotective effect under hypoxia. N2a cell lines stably expressing shRNAs that targeted Trim17 and control shRNA were constructed and treated under hypoxic condition $\left(1 \% \mathrm{O}_{2}\right)$ for $48 \mathrm{~h}$, followed by an apoptosis analysis via flow cytometry. In contrast to the increased apoptotic rate in the control cells (shCtrl), the Trim17 knock-down cells (shTrim17-1,2) showed significantly reduced apoptosis under hypoxia compared with normoxia $\left(21 \% \mathrm{O}_{2}\right)$ (fig. 5GH). As Trim 17 was shown to initiate apoptosis through the protostome-dependent degradation of Mcl-1 (Magiera, et al. 2013), we examined whether this is also the case under hypoxia. An immunoblot analysis revealed elevated Mcl1 protein level and consequently decreased active caspase-3 protein under hypoxia when compared to the control (fig. 5I). Taken together, these results suggest that by preventing MCL-1 protein from degradation, eliminating TRIM17 function confers neuroprotection against hypoxia, and therefore, probably contributes to hypoxia tolerance. In the future, further investigations addressing the linkages among the components of phenotype-genotype-fitness continuum would provide us a better understanding of gene loss and adaptation.

\section{Gene losses and PSGs cooperatively contribute to subterranean adaptations}

363 Physiological adaptations to hypoxic underground niches, such as modifications for DNA damage response (DDR) and cardiovascular system, are usually complex and expected to be involving multiple genes. We noticed that many other genomic studies had uncovered several PSGs that are also involved in DDR and heart functions in NMRs and BMRs (Kim, et al. 2011; Fang, Nevo, et al. 2014; Fang, Seim, et al. 2014; Davies, et al. 2018). Unlike gene losses, PSGs show a signature of selection that can be easily detected, and thus could be assigned as adaptive. To examine whether PSGs also show enrichments in functional groups 
that similar to EFGs in pseudogenes, we collected PSGs from previous genomic studies and compiled a PSG set for NMRs (Kim, et al. 2011; Fang, Seim, et al. 2014) and BMRs (Fang,

372 Nevo, et al. 2014; Davies, et al. 2018), respectively (see Materials and Methods).

373 Interestingly, for some of the EFGs in pseudogenes, we also observed similar functional

374 enrichments in their respective PSG sets, including functional groups related to "DNA

375 damage response", "lipid metabolism" and "neural processes" (supplementary table S8).

376 Moreover, consistent with the phenotypic convergence in heart contractile function, PSGs in

377 functional group of "cardiovascular system", for which no enrichment was observed in

378 pseudogenes, also show significant enrichment (supplementary table S8). In fact, several

379 genes involving cardiac muscle contraction in both species were under positive selection.

380 These observations highlight the collaborative contributions of not only different genes, but

381 also different types of genetic makeups to physiological adaptations. For example, protein-

382 protein interaction analysis (STRING) revealed that in both NMRs and BMRs, interaction

383 networks involving DNA damage response contain both pseudogenes and PSGs, and both of

384 them interact with other critical mediators such as ATM, ATR or TP53 (fig. 6). Such

385 simultaneous gain-of-function and lost-of-function changes in multiple genes among a

386 network reflect the strong rewiring processes in response to drastic environmental changes,

387 and rise a question of how gene losses and PSGs work together to achieve a functional

388 benefit.

389 One can easily speculate that gene losses and PSGs may have similar functional effects

390 that together result in an additive effect on a trait. For example, in the case of NMR DDR

391 network, two genes, a PSG TRIM32 and a pseudogene D7ERTD443E (FATS) interacts

392 directly with TP53 (fig. 6A). Both of them are ubiquitin ligase, but they regulate protein

393 stability of TP53 in opposite direction, with a ubiquitination-mediated stabilization by FATS

394 and a ubiquitination-mediated degradation by TRIM32 (Liu, Zhang, et al. 2014; Yan, et al.

395 2014). Pseudogenization of FATS eliminates one layer of positive control of TP53 protein

396 stability and alternatively, facilitates negative regulation together with TRIM32, or other

397 negative regulators (Vousden and Lane 2007). Another possibility considers the "sign-

398 epistatic" interaction: either types of genetic change could be initially deleterious per se,

399 while before its elimination by purifying selection, one of them may serve as the stepping

400 stone for another one to reach the otherwise inaccessible complex, beneficial functions by

401 fitness valleys crossings (Lenski, et al. 2003; Weinreich, et al. 2005; Cowperthwaite, et al. 
2006; Weinreich, et al. 2006; Covert, et al. 2013). It is also possible that losing genes serves as a compensation strategy to buffer the deleterious effects of beneficial mutations in highly pleiotropic genes or vice versa, and such compensations were recently observed experimentally in a genome-wide scale study in baker's yeast (Szamecz, et al. 2014). It is more likely that all these possible interactions exert their phenotypic effects simultaneously, due to the rewiring of a complex genetic network underlying physiological adaptations, especially to hypoxia, must have been strenuous. The roles of different types of genetic changes in complex adaptations in mammals have rarely been discussed. Our study implies the caveats of exploring the genetic mechanism of an adaptation by only looking at a single

411 type of genetic change, e.g., the PSGs. Rather, a systematic perspective with details regarding

412 the division of labour for different types of genetic makeups would be preferable.

\section{Conclusions}

415 With combination of comparative genomic approaches and in vitro experimental validations, 416 our study, for the first time, uncovered that gene losses might contribute to the evolution of 417 specialized physiological traits in both NMRs and BMRs, including altered DNA damage 418 response, specialized cardiovascular system and neuroprotection. We also highlighted that 419 the genetic mechanisms underlying physiological adaptations to subterranean niches are 420 complex, involving not only multiple genes but also different types of genetic makeups.

421 Taken together, our study provides new insights into the molecular underpinnings of 422 subterranean adaptations, highlighting the important roles of gene losses. Several 423 pseudogenes identified in BMRs and NMRs might have implications for medical researches involving aging and longevity, neuroprotection in ischemic/hypoxic injury and cardiovascular diseases. Moreover, our study suggests an integration of different genetic makeups in future studies and also has implications for the comprehensive understanding of subterranean adaptation. 


\section{Genomic data}

434 The genome sequences of each species analysed in this study were retrieved from the

435 Ensembl database (http://asia.ensembl.org/) (mouse: GRCm38.p2, release 75; rat: Rnor_6.0;

436 guinea pig: Cavpor 3.0, release 89) and NCBI database (https://www.ncbi.nlm.nih.gov/)

437 (NMR: HetGla_female_1.0; BMR: S.galili_v1.0) together with its annotated CDS and protein

438 sequences. The CDS and protein sequences from rabbit, which were used to estimate the

439 evolutionary rate, were retrieved from the Ensembl database (OryCun2.0).

\section{Identification of gene losses}

441 Basically, the gene losses in each species are defined as genes harbouring ORF-disrupting

442 mutations, including premature stop codons and/or frameshifts with intact 1:1 orthologs in

443 the mouse genome. Orthologous relationships were first established by mapping the longest

444 protein sequence for each mouse gene to each query species by simultaneously using BLAT

445 (Kent 2002) and genBlastA (She, et al. 2009). The genomic regions identified by both BLAT

446 and genBlastA were considered as orthologous sequences and were extracted with $5000 \mathrm{bp}$

447 downstream and upstream flanking sequences to predict the gene structure and ORF by

448 GeneWise (Birney, et al. 2004). Alignments containing premature stop codons and/or

449 frameshifts were identified, and the corresponding genomic regions were defined as putative

450 pseudogenic loci. To establish the unitary status of each putative pseudogenic locus, several

451 stringent filtering steps were executed as follows: 1) Loci hit by proteins belonging to large

452 gene families including olfactory receptors, zinc finger protein and vomeronasal receptors

453 were removed because of their high sequence similarity. 2) Loci hit by predicted proteins or

454 intronless cDNA/expressed sequences were removed because they are unlikely to be real or

455 unitary (Zhang, Frankish, et al. 2010). 3) To remove the potential functional redundancy, e.g.,

456 gene duplication or retrotransposition, BLAT mapping hits from the initial step were

457 reanalysed to remove loci with homologous sequences across the genome. In detail, for each

458 locus, we firstly removed hits with E-value higher than 1e-03, and then we assembled the

459 remaining hits into "copies" of this pseudogene along the mouse protein coordinates. Each

460 copy includes non-overlapping hits that together cover the mouse protein as long as possible.

461 Simply, for each "copy" with total protein length longer than $80 \%$ of the corresponding

462 mouse protein, we consider it as a potential functional redundancy and remove it. 4) We

463 examined the conserved genomic position for each locus to ensure the orthologous 
relationship. Pairwise genomic alignments between mouse and each query species were downloaded from Ensembl database. For each locus, the genomic coordinates identified by us was compared to the coordinates inferred from a pairwise genomic alignment. Only pseudogenic loci with conserved genomic position were retained for further consideration.

468 Furthermore, for genes discussed in the main text, we also manually examined the conserved gene order according to mouse genome annotation in Ensembl. To generate high-confidence pseudogene sets, false positives with disruptive mutations introduced by GeneWise, sequencing errors or annotation errors were removed through the following steps: a) in-house Perl script filtering in combination with manual inspection was performed to remove false positives introduced by GeneWise. Pseudogenes with a single disruptive mutation located at the end of the coding region (which resulted in truncated sequences longer than $90 \%$ of predicted intact protein (MacArthur 2012)), at the intron and exon boundaries or in regions with low similarity in GeneWise alignments were removed. Pseudogenes with multiple disruptions were manually inspected to remove short or low-quality GeneWise alignments. For the genes discussed in the main text, we realigned the mutated exons on mouse genome sequence using CESAR (Sharma, et al. 2017) to remove spurious disruptive mutations caused by evolutionary splice site shifts. b) to remove the sequencing and assembly errors, we retrieved the raw sequencing reads from NMRs and BMRs to check the validity of each disruption in the corresponding reads. For genes discussed in the main text, we further checked their validity using additional genomic/transcriptomic resources, i.e. raw transcriptomic reads of different tissues (Kim, et al. 2011) and genomic sequences of a male individual for NMRs (HetGla_1.0); resequencing reads of 11 individuals for BMRs (Li, et al. 2015). c) the coding potential of the corresponding genes was examined by checking the Ensembl transcriptional support level (TSL, level 1/2) and CCDS assignment, and those satisfying any one of the criteria were retained. d) pseudogenes with only two compensatory

489 frameshifts were manually examined and removed because it provided little evidence for 490 gene loss.

\section{$491 \quad$ Detection of relaxed selection}

492 Genes with disruptive mutations on coding sequence do not necessarily indicate gene

493 dispensability. Such disruptive mutations might be rare genetic variation or

494 sequencing/assembly errors. To address this issue, we detected signature of relaxed selection 495 for each pseudogene by RELAX (Wertheim, et al. 2015) and using a phylogenetic tree with 
496 rabbits as the outgroup. In detail, the coding sequences (CDS) and their translations (with

497 disruptive mutations removed) of each pseudogene were extracted from the GeneWise

498 alignments. The protein sequences were used to identify 1:1 orthologous gene in the rest of

499 the species for each pseudogene by reciprocal best BLAST hit (RBBH) method, and then, the

500 results were clustered into orthologous groups. For each orthologous group, CDS were used

501 to construct codon-based multiple sequence alignment with a phylogeny-aware alignment

502 algorithm as implemented in the PRANK program (Loytynoja and Goldman 2005). To obtain

503 reliable CDS alignments, we set up rigorous filtering criteria including: i) removing gaps and

504 ambiguous bases together with 6 bp flanking sequences with Gblocks (Castresana 2000)

505 using the default parameters; ii) removing alignment fragments with low quality (Gblocks,

506 default parameters); and iii) removing alignments with lengths of less than $150 \mathrm{bp}$.

507 Alignments containing at least two sequences were assigned to a specific tree file. In each

508 alignment, the species in which this gene loss occurred was set as "Foreground" branch and

509 the rest of species were set as "Background" branch. Only pseudogenes with a higher dN/dS

510 in foreground and a selection intensity parameter $k<1(\mathrm{k}>1$ : intensified selection; $\mathrm{k}<1$ :

511 relaxed selection) were retained.

\section{Gene ontology}

513 Orthologous mouse genes were used as surrogates to infer the functions of the pseudogenes

514 or PSGs in corresponding species. Only pseudogenes and PSGs with orthologous relationship

515 with mouse (established by BLAT and genBlastA mapping) were further considered. To

516 identify the EFGs from pseudogenes, the associated GO terms for each gene were extracted

517 from go.obo (released February 20, 2018), and the mouse gene annotation files (Mus

518 musculus, released September 29, 2017) were downloaded from the Gene Ontology

519 Consortium (http://geneontology.org/). NMRs and BMRs genes with GO terms involved in

520 certain biological processes and systems were manually clustered into functional groups

521 based on the keywords. For each functional group, we then compared the proportional

522 differences of genes from subterranean lineage pseudogene lists, subterranean lineage non-

523 pseudogene lists (with one2one mouse orthologs), control lineage pseudogene lists and

524 control lineage non-pseudogene lists (with one2one mouse orthologs). For example, A subset

525 of GO terms with a significantly higher proportion of NMR pseudogenes compared to that of

526 NMR non-pseudogenes and/or that of guinea pig pseudogenes was defined as an EFG. For

527 PSGs, EFGs were defined only when significantly higher proportion of NMR (or BMR) 
PSGs in a subset of GO terms was observed compared to that of NMR (or BMR) non-PSG background (with one2one mouse orthologs). Fisher's exact test was used to test the significances of differences in proportion and FDR-adjusted $P$-values for each multiple test were reported using p.adjust() function in R.

\section{Cell culture}

533 Primary cortical neurons were prepared from E16-E18 embryos of Kunming mice.

534 Specifically, embryonic cortices were dissected in cold DMEM (Gibco) supplemented with $53510 \%$ fetal bovine serum (Gibco) and then digested using $2 \mathrm{mg} / \mathrm{ml}$ papain (CHI). The resulting 536 cell suspension was centrifuged at $1500 \mathrm{rpm}$ for $5 \mathrm{mins}$. The cells were gently resuspended in 537 DMEM supplemented with 10\% FBS and gentamycin $(50 \mu \mathrm{g} / \mathrm{ml}$, Solarbio) and filtered 538 through a $70 \mu \mathrm{m}$ cell strainer (Corning Falcon). Then, the cells were seeded at a density of $5391.2 \times 10^{6}$ cells $/ \mathrm{ml}$ in culture dishes coated with $20 \mu \mathrm{g} / \mathrm{ml}$ poly-D-lysine (Sigma). The neurons 540 were cultured at $37^{\circ} \mathrm{C}$ in a humidified incubator with $5 \% \mathrm{CO}_{2} / 95 \%$ air, and the medium was 541 replaced 6 hours afterwards with neurobasal medium (Gibco) supplemented with B27

542 supplements (Gibco) and L-glutamine (Gibco). After 6 days in vitro (DIV 6), the neural 543 cultures were subjected to hypoxia treatment and protein extraction. N2a cells was purchased 544 from the Cell Bank of Type Culture Collection of the Chinese Academy of Sciences 545 (Shanghai; Institution Code: CBTCCCAS) and were cultured at $37^{\circ} \mathrm{C}$ in a humidified 546 incubator with 5\% $\mathrm{CO}_{2} / 95 \%$ air with MEM (Gibco) supplemented with $1.5 \mathrm{mg} / \mathrm{ml} \mathrm{NaHCO}$,

$547 \quad 0.11 \mathrm{mg} / \mathrm{ml}$ sodium pyruvate (Gibco), and 10\% FBS.

\section{Hypoxia treatment paradigms}

549 The hypoxia (1\% oxygen concentration) for the cell culture treatment was achieved 550 using a humidified hypoxia chamber (Proox, model $\mathrm{C} 21$, BioSpherix) set at $37^{\circ} \mathrm{C}, 1 \% \mathrm{O}_{2}$, 551 and $5 \% \mathrm{CO}_{2}$ and maintained with a $\mathrm{N}_{2}$ supply. The primary cortical neuron cultures and $\mathrm{N} 2 \mathrm{a}$ 552 cells (including the stable cell lines) were treated at 1\% hypoxia for various periods of time.

553 As controls, cells were cultured at $37^{\circ} \mathrm{C}$ in a humidified incubator with $5 \% \mathrm{CO}_{2}$ and $95 \%$ air.

\section{Immunoblot analysis}

555 Primary cortical neuron and N2a cells (including stable cell lines) under both hypoxic and 556 normoxic conditions were collected and lysed using RIPA buffer (Biomiga) supplemented 557 with a protease inhibitor phenylmethanesulfonyl fluoride (PMSF) and cocktail. Proteins were 558 separated by 10\% SDS-PAGE gel followed by electrotransfer to PVDF membranes 559 (Millipore). The membranes were blocked with TBST supplemented with $10 \%$ skim milk for 
30 mins at room temperature and further probed with primary antibodies at $4{ }^{\circ} \mathrm{C}$ overnight including Trim17 (1:500, Sigma); Mcl-1 (1:1000, CST); Bax (1:1000, CST); and active caspase $3(1: 500, \mathrm{CST})$ with $\beta$-actin (1:500, GeneTex) as the loading control. The membranes were subsequently incubated with HRP-conjugated secondary antibodies, and the blots were detected by ECL (Thermos Scientific). The quantifications of the protein expression levels were conducted by the gray scanning of the target proteins with Image-Pro Plus 6.0 software. Three independent experiments were performed to test the expression differences. shRNA-lentiviral infection and apoptosis detection of $\mathrm{N} 2 \mathrm{a}$ cells The HIV-derived lentiviral vector pLKO.1 containing shRNAs (TRIM17 MISSION shRNA Bacterial Glycerol Stock, Sigma) that targeted Trim17 (shTrim17-1: CCATCTGCCTTGACTACTTTA; shTrim17-2: CTGTTACCCAATTCCACTCTA) and control shRNA (shCtrl: GACACTGGGTGTGCCACAGTT) together with lentiviral packaging plasmids pCMV 8.9 and pMD2.G were co-transfected into HEK293T cells to generate lentiviruses. Supernatants containing different lentiviruses were collected 48- and 72-hours post-transfection, respectively. The N2a cells were infected with supernatants in the presence of $6 \mu \mathrm{g} / \mathrm{ml}$ polybrene for 48 hours, and $2 \mu \mathrm{g} / \mathrm{ml}$ puromycin was used to select the stable cell lines. Three stable cell lines expressing shRNAs were cultured simultaneously under 1\% hypoxia and 21\% normoxia for 48 hours and collected for FITC-conjugated annexin V (BD Pharmingen) staining followed by apoptotic cell number analysis by flow cytometry.

\section{$580 \quad$ Statistical analysis}

581 Two-tailed Fisher's exact tests were used to test the proportional differences in pseudogenes for each functional group. Paired t-tests were used to test differences in Trim17 protein levels and apoptosis rates. Hypergeometric tests

584 (http://systems.crump.ucla.edu/hypergeometric/index.php) were used to test the significance of the overlapping pseudogenes.

\section{Supplementary Materials}

588 Supplementary Table S1. Gene losses identified in each species.

589 Supplementary Table S2. Results of functional group enrichment analysis.

590 Supplementary Table S3. Mutations of pseudogenes discussed in the main text.

591 Supplementary Table S4. Independent gene losses between NMRs and BMRs. 
592 Supplementary Table S5. MGI MP information for overlapping pseudogenes.

593 Supplementary Table S6. Vision related pseudogenes.

594 Supplementary Table S7. Genetic associations of pseudogenes with cardiovascular traits.

595 Supplementary Table S8. Functional group enrichment analysis in PSGs in NMRs and

596 BMRs.

597 Supplementary Figure S1. Flowchart for identifying gene loss events in NMRs, BMRs, 598 guinea pigs and rats.

\section{Acknowledgments}

600 We thank Prof. Xu-dong Zhao, Prof. Cui-ping Yang, Dr. Dong Yang and Dr. Tao Zhang for

601 their technical support, Prof. Hua-bin Zhao for providing BMR tissue samples. We also thank

602 Dr. Michael Hiller and two anonymous reviewers for their valuable comments. This work

603 was supported by the Strategic Priority Research Program of the Chinese Academy of

604 Sciences (grant no. XDB13020400), Yunnan Province, the High-End Scientific and

605 Technological Talents program (2013HA020), and the National Natural Science Foundation

606 of China (31871277, 31321002, 31325013, and 31301013). 


\section{References}

Albalat R, Canestro C. 2016. Evolution by gene loss. Nat Rev Genet 17:379-391.

Amangyeld T, Shin YK, Lee M, Kwon B, Seo YS. 2014. Human MUS81-EME2 can cleave a variety of DNA structures including intact Holliday junction and nicked duplex. Nucleic Acids Res 42:5846-5862.

Andziak B, O'Connor TP, Qi W, DeWaal EM, Pierce A, Chaudhuri AR, Van Remmen H, Buffenstein R. 2006. High oxidative damage levels in the longest-living rodent, the naked mole-rat. Aging Cell 5:463-471.

Aoyagi T, Wada T, Kojima F, Nagai M, Harada S, Hachisu M, Murata S, Umezawa H. 1986. Inhibitors of aminopeptidase B suppress the development of hypertension in spontaneously hypertensive rats. Chem Pharm Bull (Tokyo) 34:4852-4854.

Arieli R, Ar A. 1981. Heart-Rate Responses of the Mole Rat (Spalax-Ehrenbergi) in Hypercapnic, Hypoxic, and Cold Conditions. Physiol Zool 54:14-21.

Ashur-Fabian O, Avivi A, Trakhtenbrot L, Adamsky K, Cohen M, Kajakaro G, Joel A, Amariglio N, Nevo E, Rechavi G. 2004. Evolution of p53 in hypoxia-stressed Spalax mimics human tumor mutation. Proc Natl Acad Sci U S A 101:12236-12241.

Aurich-Costa J, Cadel S, Gouzy C, Foulon T, Cherif D, Cohen P. 1997. Assignment of the aminopeptidase B gene (RNPEP) to human chromosome 1 band $\mathrm{q} 32$ by in situ hybridization. Cytogenet Cell Genet 79:143-144.

Avivi A, Albrecht U, Oster H, Joel A, Beiles A, Nevo E. 2001. Biological clock in total darkness: the Clock/MOP3 circadian system of the blind subterranean mole rat. Proc Natl Acad Sci U S A 98:13751-13756.

Avivi A, Gerlach F, Joel A, Reuss S, Burmester T, Nevo E, Hankeln T. 2010. Neuroglobin, cytoglobin, and myoglobin contribute to hypoxia adaptation of the subterranean mole rat Spalax. Proc Natl Acad Sci U S A 107:21570-21575.

Barzilai A, Yamamoto K. 2004. DNA damage responses to oxidative stress. DNA Repair (Amst) 3:1109-1115.

Bhat KP, Krishnamoorthy A, Dungrawala H, Garcin EB, Modesti M, Cortez D. 2018. RADX Modulates RAD51 Activity to Control Replication Fork Protection. Cell Rep 24:538-545. Birney E, Clamp M, Durbin R. 2004. GeneWise and Genomewise. Genome Res 14:988-995. Bonduriansky R, Day T. 2009. Nongenetic inheritance and its evolutionary implications. Annual Review of Ecology, Evolution, and Systematics 40:103-125. 
Borlaug BA, Melenovsky V, Marhin T, Fitzgerald P, Kass DA. 2005. Sildenafil inhibits betaadrenergic-stimulated cardiac contractility in humans. Circulation 112:2642-2649.

Buffenstein R, Woodley R, Thomadakis C, Daly TJ, Gray DA. 2001. Cold-induced changes in thyroid function in a poikilothermic mammal, the naked mole-rat. Am J Physiol Regul Integr Comp Physiol 280:R149-155.

Caballero B, Tomas-Zapico C, Vega-Naredo I, Sierra V, Tolivia D, Hardeland R, RodriguezColunga MJ, Joel A, Nevo E, Avivi A, et al. 2006. Antioxidant activity in Spalax ehrenbergi: a possible adaptation to underground stress. J Comp Physiol A Neuroethol Sens Neural Behav Physiol 192:753-759.

Castresana J. 2000. Selection of conserved blocks from multiple alignments for their use in phylogenetic analysis. Mol Biol Evol 17:540-552.

Choo SW, Rayko M, Tan TK, Hari R, Komissarov A, Wee WY, Yurchenko AA, Kliver S, Tamazian G, Antunes A, et al. 2016. Pangolin genomes and the evolution of mammalian scales and immunity. Genome Res 26:1312-1322.

Covert AW, 3rd, Lenski RE, Wilke CO, Ofria C. 2013. Experiments on the role of deleterious mutations as stepping stones in adaptive evolution. Proc Natl Acad Sci U S A 110:E31713178 .

Cowperthwaite MC, Bull JJ, Meyers LA. 2006. From bad to good: Fitness reversals and the ascent of deleterious mutations. PLoS Comput Biol 2:e141.

Davies KTJ, Bennett NC, Faulkes CG, Rossiter SJ. 2018. Limited Evidence for Parallel Molecular Adaptations Associated with the Subterranean Niche in Mammals: A Comparative Study of Three Superorders. Mol Biol Evol 35:2544-2559.

Davis-Walton J, Sherman PW. (Davis-Walton1994 co-authors). 1994. Sleep arrhythmia in the eusocial naked mole-rat. Naturwissenschaften 81:272-275.

Delaney MA, Ward JM, Walsh TF, Chinnadurai SK, Kerns K, Kinsel MJ, Treuting PM. 2016. Initial Case Reports of Cancer in Naked Mole-rats (Heterocephalus glaber). Vet Pathol 53:691-696.

Deming JD, Pak JS, Brown BM, Kim MK, Aung MH, Eom YS, Shin JA, Lee EJ, Pardue MT, Craft CM. 2015. Visual Cone Arrestin 4 Contributes to Visual Function and Cone Health. Invest Ophthalmol Vis Sci 56:5407-5416.

Deng X, Sabino EC, Cunha-Neto E, Ribeiro AL, Ianni B, Mady C, Busch MP, Seielstad M. 2013. Genome wide association study (GWAS) of Chagas cardiomyopathy in 
Trypanosoma cruzi seropositive subjects. Plos One 8.

Domankevich V, Eddini H, Odeh A, Shams I. 2018. Resistance to DNA damage and enhanced DNA repair capacity in the hypoxia-tolerant blind mole rat Spalax carmeli. J Exp Biol 221. Drane P, Brault ME, Cui G, Meghani K, Chaubey S, Detappe A, Parnandi N, He Y, Zheng XF, Botuyan MV, et al. 2017. TIRR regulates 53BP1 by masking its histone methyl-lysine binding function. Nature 543:211-216.

Du K, Yang L, He S. 2015. Phylogenomic analyses reveal a molecular signature linked to subterranean adaptation in rodents. Bmc Evol Biol 15:287.

Dungrawala H, Bhat KP, Le Meur R, Chazin WJ, Ding X, Sharan SK, Wessel SR, Sathe AA, Zhao R, Cortez D. 2017. RADX Promotes Genome Stability and Modulates Chemosensitivity by Regulating RAD51 at Replication Forks. Mol Cell 67:374-386 e375.

Emerling CA. 2018. Regressed but Not Gone: Patterns of Vision Gene Loss and Retention in Subterranean Mammals. Integr Comp Biol 58:441-451.

Emerling CA, Springer MS. 2014. Eyes underground: regression of visual protein networks in subterranean mammals. Mol Phylogenet Evol 78:260-270.

Evangelou E, Warren HR, Mosen-Ansorena D, Mifsud B, Pazoki R, Gao H, Ntritsos G, Dimou N, Cabrera CP, Karaman I. 2018. Genetic analysis of over 1 million people identifies 535 new loci associated with blood pressure traits. Nat Genet 50:1412-1425.

Fang X, Nevo E, Han L, Levanon EY, Zhao J, Avivi A, Larkin D, Jiang X, Feranchuk S, Zhu Y, et al. 2014. Genome-wide adaptive complexes to underground stresses in blind mole rats Spalax. Nat Commun 5:3966.

Fang X, Seim I, Huang Z, Gerashchenko MV, Xiong Z, Turanov AA, Zhu Y, Lobanov AV, Fan D, Yim SH, et al. 2014. Adaptations to a subterranean environment and longevity revealed by the analysis of mole rat genomes. Cell Rep 8:1354-1364.

Feitosa MF, Kraja AT, Chasman DI, Sung YJ, Winkler TW, Ntalla I, Guo X, Franceschini N, Cheng C-Y, Sim X. 2018. Novel genetic associations for blood pressure identified via gene-alcohol interaction in up to $570 \mathrm{~K}$ individuals across multiple ancestries. Plos One 13.

Gan P, Patterson M, Velasquez A, Wang K, Tian D, Windle JJ, Tao G, Judge DP, Makita T, Park TJ, et al. 2019. Tnni3k alleles influence ventricular mononuclear diploid cardiomyocyte frequency. PLoS Genet 15:e1008354.

Giannetta E, Isidori AM, Galea N, Carbone I, Mandosi E, Vizza CD, Naro F, Morano S, Fedele F, Lenzi A. 2012. Chronic Inhibition of cGMP phosphodiesterase 5A improves diabetic 
cardiomyopathy: a randomized, controlled clinical trial using magnetic resonance imaging with myocardial tagging. Circulation 125:2323-2333.

Giri A, Hellwege JN, Keaton JM, Park J, Qiu C, Warren HR, Torstenson ES, Kovesdy CP, Sun YV, Wilson OD. 2019. Trans-ethnic association study of blood pressure determinants in over 750,000 individuals. Nat Genet 51:51-62.

Gorbunova V, Hine C, Tian X, Ablaeva J, Gudkov AV, Nevo E, Seluanov A. 2012. Cancer resistance in the blind mole rat is mediated by concerted necrotic cell death mechanism. Proc Natl Acad Sci U S A 109:19392-19396.

Gottlieb RA, Burleson KO, Kloner RA, Babior BM, Engler RL. 1994. Reperfusion injury induces apoptosis in rabbit cardiomyocytes. J Clin Invest 94:1621-1628.

Grimes KM, Reddy AK, Lindsey ML, Buffenstein R. 2014. And the beat goes on: maintained cardiovascular function during aging in the longest-lived rodent, the naked mole-rat. Am J Physiol Heart Circ Physiol 307:H284-291.

Grimes KM, Voorhees A, Chiao YA, Han HC, Lindsey ML, Buffenstein R. 2014. Cardiac function of the naked mole-rat: ecophysiological responses to working underground. Am J Physiol Heart Circ Physiol 306:H730-737.

Hecker N, Sharma V, Hiller M. 2019. Convergent gene losses illuminate metabolic and physiological changes in herbivores and carnivores. Proc Natl Acad Sci U S A 116:30363041 .

Huelsmann M, Hecker N, Springer MS, Gatesy J, Sharma V, Hiller M. 2019. Genes lost during the transition from land to water in cetaceans highlight genomic changes associated with aquatic adaptations. Science advances 5:eaaw6671.

Jarvis JU. 1981. Eusociality in a mammal: cooperative breeding in naked mole-rat colonies. Science 212:571-573.

Kent WJ. 2002. BLAT--the BLAST-like alignment tool. Genome Res 12:656-664.

Kim EB, Fang X, Fushan AA, Huang Z, Lobanov AV, Han L, Marino SM, Sun X, Turanov AA, Yang P, et al. 2011. Genome sequencing reveals insights into physiology and longevity of the naked mole rat. Nature 479:223-227.

Kimchi T, Terkel J. 2001. Magnetic compass orientation in the blind mole rat Spalax ehrenbergi. J Exp Biol 204:751-758.

Lassot I, Robbins I, Kristiansen M, Rahmeh R, Jaudon F, Magiera MM, Mora S, Vanhille L, Lipkin A, Pettmann B, et al. 2010. Trim17, a novel E3 ubiquitin-ligase, initiates neuronal 
apoptosis. Cell Death Differ 17:1928-1941.

Lenski RE, Ofria C, Pennock RT, Adami C. 2003. The evolutionary origin of complex features. Nature 423:139-144.

Li K, Hong W, Jiao H, Wang GD, Rodriguez KA, Buffenstein R, Zhao Y, Nevo E, Zhao H. 2015. Sympatric speciation revealed by genome-wide divergence in the blind mole rat Spalax. Proc Natl Acad Sci U S A 112:11905-11910.

Li Z, Zhang Q, Mao JH, Weise A, Mrasek K, Fan X, Zhang X, Liehr T, Lu KH, Balmain A, et al. 2010. An HDAC1-binding domain within FATS bridges p21 turnover to radiationinduced tumorigenesis. Oncogene 29:2659-2671.

Liu J, Zhang C, Wang XL, Ly P, Belyi V, Xu-Monette ZY, Young KH, Hu W, Feng Z. 2014. E3 ubiquitin ligase TRIM32 negatively regulates tumor suppressor p53 to promote tumorigenesis. Cell Death Differ 21:1792-1804.

Liu X, Gao Y, Lu Y, Zhang J, Li L, Yin F. 2014. Downregulation of NEK11 is associated with drug resistance in ovarian cancer. Int J Oncol 45:1266-1274.

Liu Z, Wang W, Zhang TZ, Li GH, He K, Huang JF, Jiang XL, Murphy RW, Shi P. 2014. Repeated functional convergent effects of NaV1.7 on acid insensitivity in hibernating mammals. Proc Biol Sci 281:20132950.

Long E, Zhang J. 2020. Natural selection contributes to the myopia epidemic. National Science Review.

Loytynoja A, Goldman N. 2005. An algorithm for progressive multiple alignment of sequences with insertions. Proc Natl Acad Sci U S A 102:10557-10562.

MacArthur DG. 2012. A systematic survey of loss-of-function variants in human proteincoding genes (vol 335, pg 823, 2012). Science 336:296-296.

MacArthur J, Bowler E, Cerezo M, Gil L, Hall P, Hastings E, Junkins H, McMahon A, Milano A, Morales J. 2017. The new NHGRI-EBI Catalog of published genome-wide association studies (GWAS Catalog). Nucleic acids research 45:D896-D901.

Magiera MM, Mora S, Mojsa B, Robbins I, Lassot I, Desagher S. 2013. Trim17-mediated ubiquitination and degradation of Mcl-1 initiate apoptosis in neurons. Cell Death Differ 20:281-292.

Malik A, Korol A, Hubner S, Hernandez AG, Thimmapuram J, Ali S, Glaser F, Paz A, Avivi A, Band M. 2011. Transcriptome sequencing of the blind subterranean mole rat, Spalax galili: utility and potential for the discovery of novel evolutionary patterns. Plos One 
6:e21227.

Malik A, Korol A, Weber M, Hankeln T, Avivi A, Band M. 2012. Transcriptome analysis of the spalax hypoxia survival response includes suppression of apoptosis and tight control of angiogenesis. BMC Genomics 13:615.

Mann MD, Rehkamper G, Reinke H, Frahm HD, Necker R, Nevo E. 1997. Size of somatosensory cortex and of somatosensory thalamic nuclei of the naturally blind mole rat, Spalax ehrenbergi. J Hirnforsch 38:47-59.

Melixetian M, Klein DK, Sorensen CS, Helin K. 2009. NEK11 regulates CDC25A degradation and the IR-induced G2/M checkpoint. Nat Cell Biol 11:1247-1253.

Meyer WK, Jamison J, Richter R, Woods SE, Partha R, Kowalczyk A, Kronk C, Chikina M, Bonde RK, Crocker DE, et al. 2018. Ancient convergent losses of Paraoxonase 1 yield potential risks for modern marine mammals. Science 361:591-594.

Mitchell TW, Buffenstein R, Hulbert AJ. 2007. Membrane phospholipid composition may contribute to exceptional longevity of the naked mole-rat (Heterocephalus glaber): a comparative study using shotgun lipidomics. Exp Gerontol 42:1053-1062.

Nasser NJ, Nevo E, Shafat I, Ilan N, Vlodavsky I, Avivi A. 2005. Adaptive evolution of heparanase in hypoxia-tolerant Spalax: gene cloning and identification of a unique splice variant. Proc Natl Acad Sci U S A 102:15161-15166.

Nevo E. 1979. Adaptive Convergence and Divergence of Subterranean Mammals. Annual Review of Ecology and Systematics 10:269-308.

Olson MV. 1999. When less is more: gene loss as an engine of evolutionary change. Am J Hum Genet 64:18-23.

Park TJ, Reznick J, Peterson BL, Blass G, Omerbasic D, Bennett NC, Kuich P, Zasada C, Browe BM, Hamann W, et al. 2017. Fructose-driven glycolysis supports anoxia resistance in the naked mole-rat. Science 356:307-311.

Patterson M, Barske L, Van Handel B, Rau CD, Gan P, Sharma A, Parikh S, Denholtz M, Huang Y, Yamaguchi Y, et al. 2017. Frequency of mononuclear diploid cardiomyocytes underlies natural variation in heart regeneration. Nat Genet 49:1346-1353.

Pepe A, West SC. 2014. Substrate specificity of the MUS81-EME2 structure selective endonuclease. Nucleic Acids Res 42:3833-3845.

Perez NG, Piaggio MR, Ennis IL, Garciarena CD, Morales C, Escudero EM, Cingolani OH, Chiappe de Cingolani G, Yang XP, Cingolani HE. 2007. Phosphodiesterase 5A inhibition 
induces $\mathrm{Na}+\mathrm{H}+$ exchanger blockade and protection against myocardial infarction. Hypertension 49:1095-1103.

Perez VI, Buffenstein R, Masamsetti V, Leonard S, Salmon AB, Mele J, Andziak B, Yang T,

Edrey Y, Friguet B, et al. 2009. Protein stability and resistance to oxidative stress are determinants of longevity in the longest-living rodent, the naked mole-rat. Proc Natl Acad Sci U S A 106:3059-3064.

Petruseva IO, Evdokimov AN, Lavrik OI. 2017. Genome Stability Maintenance in Naked Mole-Rat. Acta Naturae 9:31-41.

Prudent X, Parra G, Schwede P, Roscito JG, Hiller M. 2016. Controlling for Phylogenetic Relatedness and Evolutionary Rates Improves the Discovery of Associations Between Species' Phenotypic and Genomic Differences. Mol Biol Evol 33:2135-2150.

Qian W, Liao BY, Chang AY, Zhang J. 2010. Maintenance of duplicate genes and their functional redundancy by reduced expression. Trends Genet 26:425-430.

Ramírez J, van Duijvenboden S, Ntalla I, Mifsud B, Warren HR, Tzanis E, Orini M, Tinker A, Lambiase PD, Munroe PB. 2018. Thirty loci identified for heart rate response to exercise and recovery implicate autonomic nervous system. Nat Commun 9:1-10.

Ramirez JM, Folkow LP, Blix AS. 2007. Hypoxia tolerance in mammals and birds: from the wilderness to the clinic. Annu Rev Physiol 69:113-143.

Riccio AP, Goldman BD. 2000. Circadian rhythms of locomotor activity in naked mole-rats (Heterocephalus glaber). Physiol Behav 71:1-13.

Seluanov A, Hine C, Azpurua J, Feigenson M, Bozzella M, Mao Z, Catania KC, Gorbunova V. 2009. Hypersensitivity to contact inhibition provides a clue to cancer resistance of naked mole-rat. Proc Natl Acad Sci U S A 106:19352-19357.

Shams I, Avivi A, Nevo E. 2004. Hypoxic stress tolerance of the blind subterranean mole rat: expression of erythropoietin and hypoxia-inducible factor 1 alpha. Proc Natl Acad Sci U S A 101:9698-9703.

Shao Y, Li JX, Ge RL, Zhong L, Irwin DM, Murphy RW, Zhang YP. 2015. Genetic adaptations of the plateau zokor in high-elevation burrows. Sci Rep 5:17262.

Sharma V, Hecker N, Roscito JG, Foerster L, Langer BE, Hiller M. 2018. A genomics approach reveals insights into the importance of gene losses for mammalian adaptations. Nat Commun 9:1215.

Sharma V, Schwede P, Hiller M. 2017. CESAR 2.0 substantially improves speed and accuracy 
of comparative gene annotation. Bioinformatics 33:3985-3987.

She R, Chu JS, Wang K, Pei J, Chen N. 2009. GenBlastA: enabling BLAST to identify homologous gene sequences. Genome Res 19:143-149.

Smith ES, Omerbasic D, Lechner SG, Anirudhan G, Lapatsina L, Lewin GR. 2011. The molecular basis of acid insensitivity in the African naked mole-rat. Science 334:1557-1560.

Szamecz B, Boross G, Kalapis D, Kovacs K, Fekete G, Farkas Z, Lazar V, Hrtyan M, Kemmeren P, Groot Koerkamp MJ, et al. 2014. The genomic landscape of compensatory evolution. PLoS Biol 12:e1001935.

Takimoto E, Champion HC, Li M, Belardi D, Ren S, Rodriguez ER, Bedja D, Gabrielson KL, Wang Y, Kass DA. 2005. Chronic inhibition of cyclic GMP phosphodiesterase 5A prevents and reverses cardiac hypertrophy. Nat Med 11:214-222.

Techer H, Koundrioukoff S, Carignon S, Wilhelm T, Millot GA, Lopez BS, Brison O, Debatisse M. 2016. Signaling from Mus81-Eme2-Dependent DNA Damage Elicited by Chk1 Deficiency Modulates Replication Fork Speed and Origin Usage. Cell Rep 14:1114-1127. Tedford RJ, Hemnes AR, Russell SD, Wittstein IS, Mahmud M, Zaiman AL, Mathai SC, Thiemann DR, Hassoun PM, Girgis RE, et al. 2008. PDE5A inhibitor treatment of persistent pulmonary hypertension after mechanical circulatory support. Circ Heart Fail $1: 213-219$.

Tian X, Azpurua J, Hine C, Vaidya A, Myakishev-Rempel M, Ablaeva J, Mao Z, Nevo E, Gorbunova V, Seluanov A. 2013. High-molecular-mass hyaluronan mediates the cancer resistance of the naked mole rat. Nature 499:346-349.

Vagnozzi RJ, Gatto GJ, Jr., Kallander LS, Hoffman NE, Mallilankaraman K, Ballard VL, Lawhorn BG, Stoy P, Philp J, Graves AP, et al. 2013. Inhibition of the cardiomyocytespecific kinase TNNI3K limits oxidative stress, injury, and adverse remodeling in the ischemic heart. Sci Transl Med 5:207ra141.

Vavouri T, Semple JI, Lehner B. 2008. Widespread conservation of genetic redundancy during a billion years of eukaryotic evolution. Trends Genet 24:485-488.

Vousden KH, Lane DP. 2007. p53 in health and disease. Nat Rev Mol Cell Biol 8:275-283.

Wang H, Wang L, Song L, Zhang YW, Ye J, Xu RX, Shi N, Meng XM. 2013. TNNI3K is a novel mediator of myofilament function and phosphorylates cardiac troponin I. Braz J Med Biol Res 46:128-137.

Wang X, Wang J, Su M, Wang C, Chen J, Wang H, Song L, Zou Y, Zhang L, Zhang Y, et al. 
2013. TNNI3K, a cardiac-specific kinase, promotes physiological cardiac hypertrophy in transgenic mice. Plos One 8:e58570.

Weber RE, Heath ME, White FN. 1986. Oxygen binding functions of blood and hemoglobin from the Chinese pangolin, Manis pentadactyla: possible implications of burrowing and low body temperature. Respir Physiol 64:103-112.

Weinreich DM, Delaney NF, Depristo MA, Hartl DL. 2006. Darwinian evolution can follow only very few mutational paths to fitter proteins. Science 312:111-114.

Weinreich DM, Watson RA, Chao L. 2005. Perspective: Sign epistasis and genetic constraint on evolutionary trajectories. Evolution 59:1165-1174.

Wertheim JO, Murrell B, Smith MD, Kosakovsky Pond SL, Scheffler K. 2015. RELAX: detecting relaxed selection in a phylogenetic framework. Mol Biol Evol 32:820-832.

Widmer HR, Hoppeler H, Nevo E, Taylor CR, Weibel ER. 1997. Working underground: respiratory adaptations in the blind mole rat. Proc Natl Acad Sci U S A 94:2062-2067.

Wittkopp PJ, Kalay G. 2012. Cis-regulatory elements: molecular mechanisms and evolutionary processes underlying divergence. Nat Rev Genet 13:59-69.

Yan S, Qiu L, Ma K, Zhang X, Zhao Y, Zhang J, Li X, Hao X, Li Z. 2014. FATS is an E2independent ubiquitin ligase that stabilizes p53 and promotes its activation in response to DNA damage. Oncogene 33:5424-5433.

Yellon DM, Hausenloy DJ. 2007. Myocardial reperfusion injury. N Engl J Med 357:1121-1135.

Zhang X, Zhang Q, Zhang J, Qiu L, Yan SS, Feng J, Sun Y, Huang X, Lu KH, Li Z. 2010. FATS is a transcriptional target of p53 and associated with antitumor activity. Mol Cancer $9: 244$.

Zhang ZD, Frankish A, Hunt T, Harrow J, Gerstein M. 2010. Identification and analysis of unitary pseudogenes: historic and contemporary gene losses in humans and other primates. Genome Biol 11:R26.

Zhao Y, Meng XM, Wei YJ, Zhao XW, Liu DQ, Cao HQ, Liew CC, Ding JF. 2003. Cloning and characterization of a novel cardiac-specific kinase that interacts specifically with cardiac troponin I. J Mol Med (Berl) 81:297-304.

Zhao Y, Tyshkovskiy A, Munoz-Espin D, Tian X, Serrano M, de Magalhaes JP, Nevo E, Gladyshev VN, Seluanov A, Gorbunova V. 2018. Naked mole rats can undergo developmental, oncogene-induced and DNA damage-induced cellular senescence. Proc Natl Acad Sci U S A 115:1801-1806. 
bioRxiv preprint doi: https://doi.org/10.1101/2021.05.28.446201; this version posted May 29, 2021. The copyright holder for this preprint

(which was not certified by peer review) is the author/funder. All rights reserved. No reuse allowed without permission. 
Table 1. Statistics of each step of identifying gene loss events in NMRs, BMRs, guinea pigs and rats.

\begin{tabular}{llllll}
\hline Species & $\begin{array}{l}\text { Overlapping } \\
\text { best hits }\end{array}$ & $\begin{array}{l}\text { Putative } \\
\text { pseudogenic } \\
\text { loci }\end{array}$ & $\begin{array}{l}\text { Unitary } \\
\text { pseudogenic } \\
\text { loci }\end{array}$ & $\begin{array}{l}\text { False } \\
\text { positives } \\
\text { removed }\end{array}$ & $\begin{array}{l}\text { Selective } \\
\text { constraints } \\
\text { relaxed }\end{array}$ \\
\hline NMRs & 14254 & 1362 & 883 & 211 & 167 \\
BMRs & 16546 & 1609 & 1023 & 211 & 139 \\
Guinean pigs & 15969 & 2695 & 2217 & $617^{*}$ & 341 \\
Rats & 17396 & 2821 & 1701 & $389^{*}$ & 112 \\
\hline
\end{tabular}

*Sequencing errors were not examined.

\section{Figure legends}

Fig. 1. Gene losses identified in each species. The numbers of gene losses were labelled above each branch of the phylogenetic tree of species investigated.

Fig. 2. Functional groups enrichment analysis. Linage-specific and shared EFGs identified in NMR (A) and BMR (B) pseudogenes. The proportions of pseudogenes and functional genes background from each species in each EFG are shown and proportional difference in each EFG were tested with Fisher's exact test. $* P<0.05, * * P<0.01, * * * P<0.001, * * * * P<0.0001$.

Fig. 3. Functional convergences of gene losses between NMRs and BMRs in DDR pathway and cardiac features. (A) A part of DNA damage response pathway is represented. (B) Schematic diagrams of cardiac adaptations in NMRs and BMRs. Genes highlighted in green and blue are gene losses from NMRs and BMRs, respectively, and the gene highlighted in orange indicates shared gene loss between NMRs and BMRs.

Fig. 4. Convergent gene losses in species pairs from similar and distinct environments. (A) Proportional difference of overlapping pseudogenes among 4 species pairs including NMRsBMRs (N-B), guinea pigs-rats (G-R), NMRs-rats (N-R) and BMRs-guinea pigs (B-G). The numbers of overlapping and total pseudogene from each pair are labelled above the bars. $P$ values are from two-tailed Fisher's exact test. (B) Venn diagram showing the one-to-one orthologous genes (large box) and overlapping pseudogenes in NMRs-BMRs pair. $P$-value 
from a hypergeometric test indicates that gene losses in NMRs and BMRs are not independent from each other.

Fig. 5. Functional tests of TRIM17 for its role in hypoxia adaptation. (A) Coding sequence alignment of TRIM17 orthologous genes from mouse, NMR, BMR and 7 other mammals. One stop codon in NMRs and one stop codon/4 frameshifts in BMRs were observed that resulted in disrupted coding potential of TRIM17 gene. (B to D) Examples of inactivating mutations in toothed whales (B), baleen whales (C) and pangolins (D). The positions of all mutations shown in this figure are labelled under the corresponding alignment using mouse Trim17 coding sequence as reference. (E to F) Immunoblot analyses for TRIM17 protein level changes during hypoxia treatments in primary cortical neurons $(\mathbf{E})$ and N2a cell lines $(\mathbf{F})$. "+" indicates hypoxia treatment and "-" indicates normoxia treatment. Quantification and relative foldchange analysis of protein level showed a significantly decrease of Trim 17 protein induced by hypoxia in both primary cortical neurons and N2a cells. Data from three independent assays were used to perform paired two-tailed t-test, ${ }^{*} P<0.05$. (G to I) Knockdown of Trim17 expression by shRNAs (shTRIM17-1 and shTRIM17-2) provides neuroprotection under hypoxia $\left(1 \% \mathrm{O}_{2}\right)$ compared to control (shCtrl). Apoptotic cells are detected by staining cells with PI and Annexin V-FITC followed by flowmetry analysis. Cells with Trim17 knockdown showed significantly lower apoptosis rate under hypoxia compared to control, while no significant changes were observed under nomorxia $(\mathbf{G}, \mathbf{H})$, paired two-tail t-test, $* P<0.05$, ${ }^{* *} P<0.01$. Immunoblot analysis for corresponding cells shows elevated Mcl-1 protein level and decreased activated caspase-3 protein level under hypoxia in cells with Trim 17 knockdown (I).

Fig. 6. Interacting network of DDR related proteins. Different genes with different types of genetic changes are interacting with each other in both NMRs (A) and BMRs (B). PSGs are highlighted in green and gene losses are highlighted in yellow, and core mediators of DDR are highlighted in black. Interaction information were derived from STRING database (https://string-db.org/, Version 11.0). 
Mouse

112 Rat

139

Blind mole rat

341

Guinea pig

167

Naked mole rat

\begin{tabular}{llll}
\hline & 1 & 1 & 1
\end{tabular}$\quad$ Million years(mya)


A

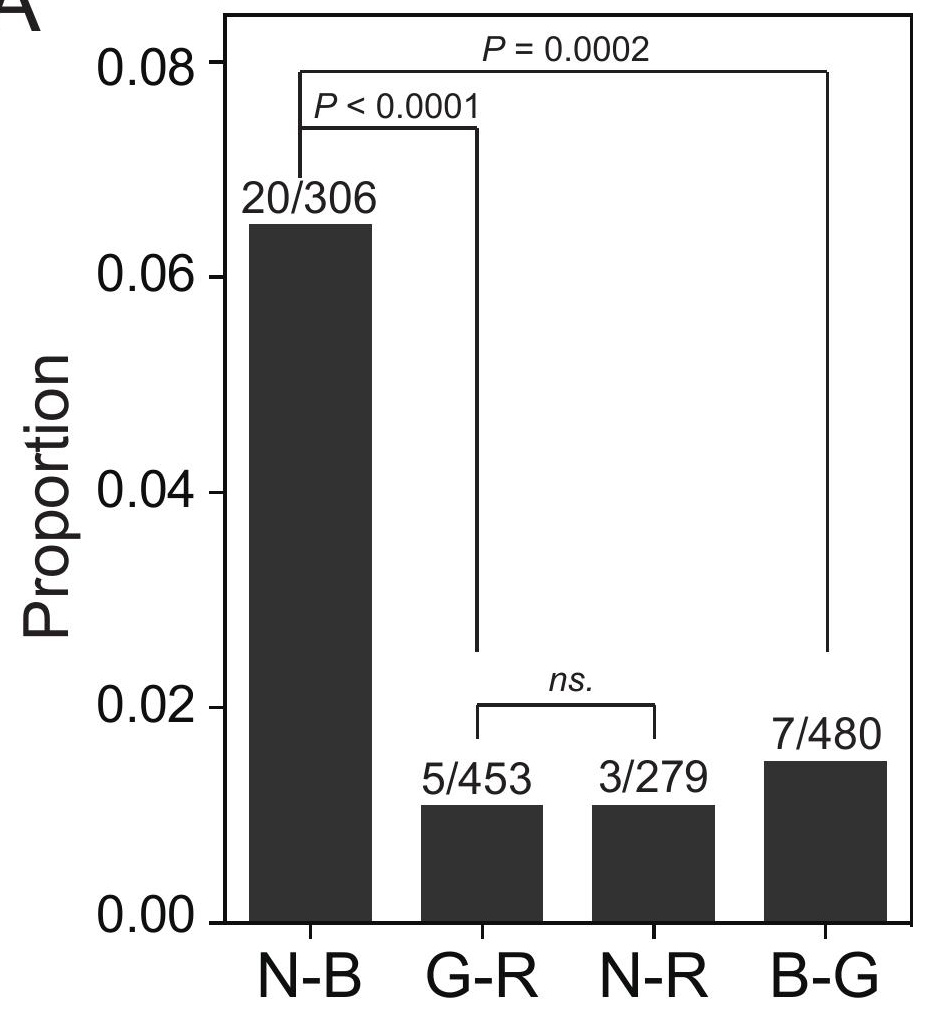

B

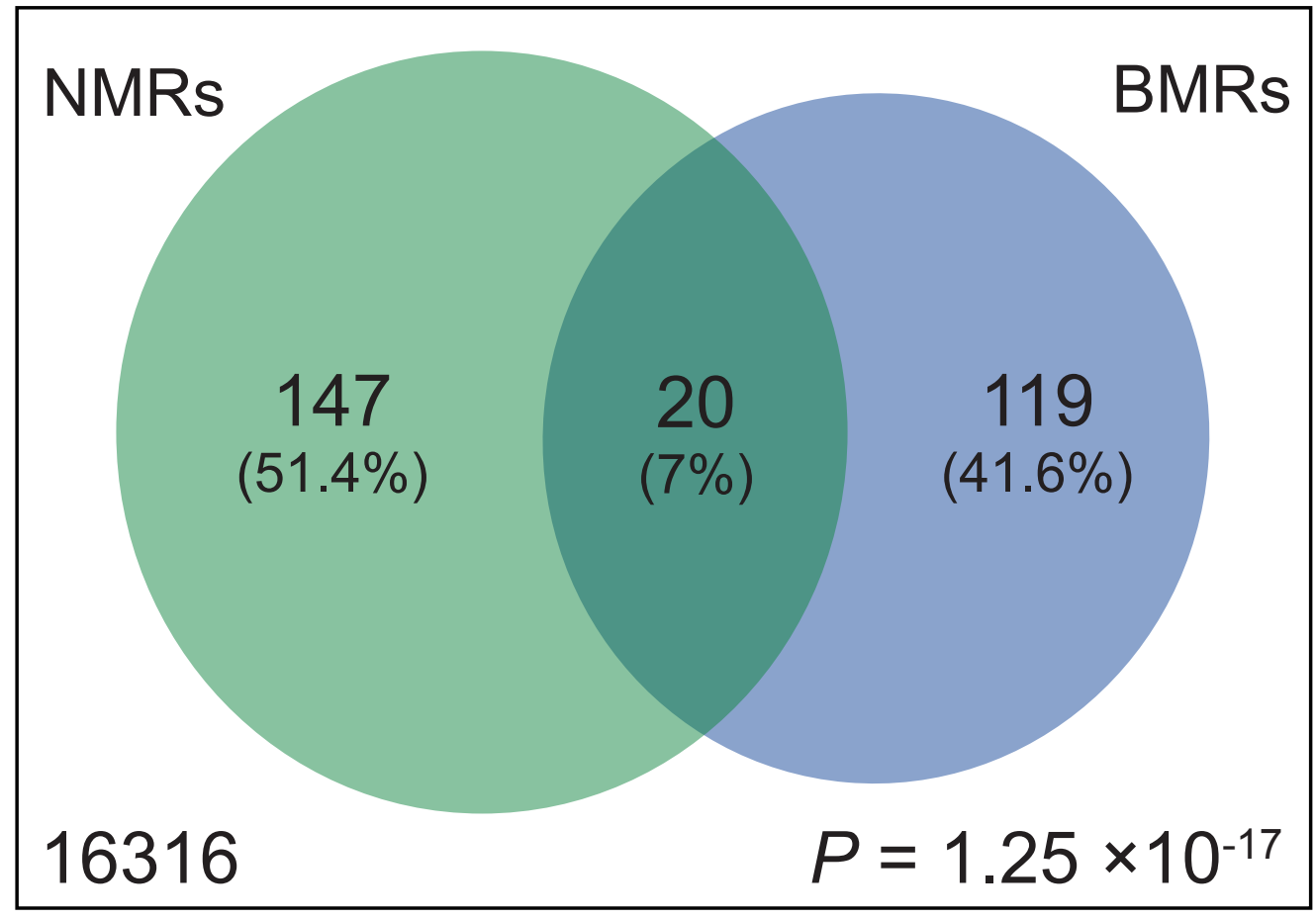




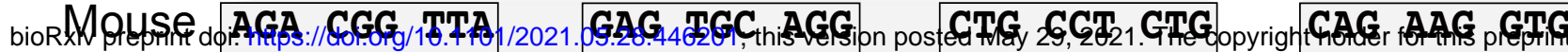

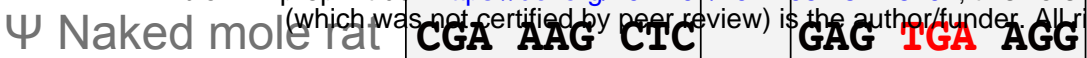
$\Psi$ Blind mole rat $\mathbf{A G A}$-GG TTG Guinea pig AGA AAG CTG Rat AGA CGG TTG Rabbit AGA AAG CTG

Pig AGA AAA CTG

Dog AGA AAG CTG Hedgehog AGA AAG CTG Armadillo AGA AAG CTG bp deletion GAG TGC AGG GAG TGC AGG GAG TGC AGG GAG TGC AGG GAA TGC CGT GAG TGT CGC GAG TGC CGC GAG TGC AGG C.193-195 Stop codon

\begin{tabular}{|c|c|c|}
\hline 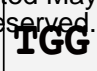 & 'Cct & \\
\hline CTG & T-T & GTC \\
\hline ITG & $\mathrm{CCT}$ & GTC \\
\hline TTG & ССT & CTI \\
\hline CTG & $\operatorname{ccc}$ & GTC \\
\hline GTC & ССT & $\mathrm{ATT}$ \\
\hline GTC & CCC & ATC \\
\hline CTC & $\operatorname{ccc}$ & GT \\
\hline CTC & CCG & \\
\hline
\end{tabular}
GAG CAG A-G GTG CAG AAG GTG GAG AAG ATG GAG AAG ATG GAG AAG ATG CGC AAG GCC GAG AAG ATG C.572 $1 \mathrm{bp}$ deletion GAG AAA GTG

\section{C}

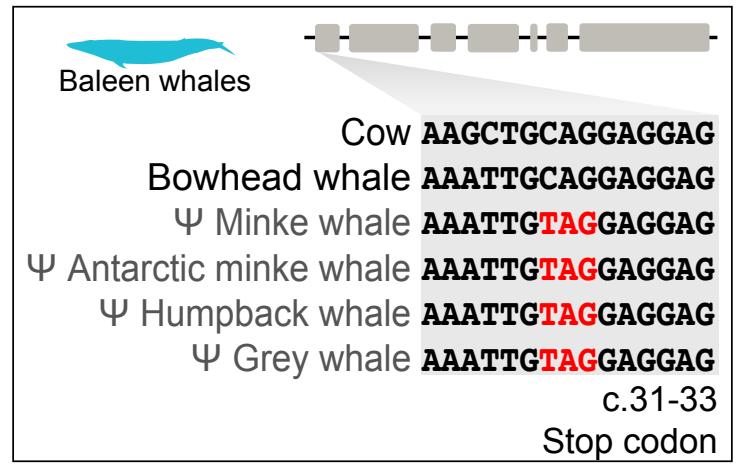

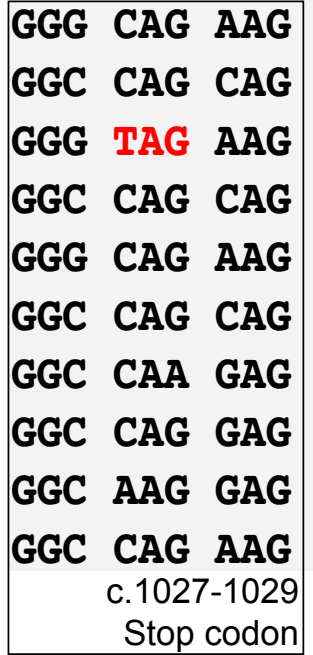

GGG CAG AAG GGC CAG CAG GGG TAG AAG GGC CAG CAG GGG CAG AAG GGC CAG CAG GGC CAA GAG GGC CAG GAG GGC AAG GAG Stop codon

\begin{tabular}{|lll|}
\hline GGA & AGG & CAC \\
GGG & AGG & CTC \\
--- & --- & $--C$ \\
GGA & AAG & CAC \\
GGA & AGG & CAC \\
GGG & CGG & CAC \\
GGG & AGG & CAC \\
GGA & AGG & CAC \\
GGG & CGC & CAC \\
GGG & AGA & CAC \\
C.1045-1052 \\
8bp deletion \\
\hline
\end{tabular}

B
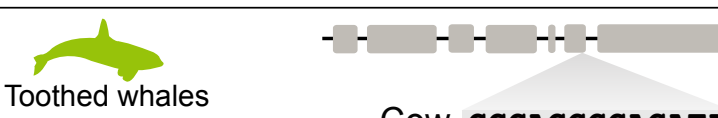

COW CCCAGGGCAGATA

Bowhead whale GCCGGGGCAGATA

$\Psi$ Yangtze finless porpoise GCTGGG-CAGATA $\Psi$ Narwhal GCCGGG-CAGATA

$\Psi$ Beluga whale GCCGGG-CAGATA $\Psi$ Common bottlenose dolphin GCCGGG-CAGATA $\Psi$ Killer whale GCCGGG-CAGATA $\Psi$ Pacific white-sided dolphin GCCGGG-CAGATA $\Psi$ Long-finned pilot whale GCCGGG-CAGATA c.855:1bp deletion

\section{D}

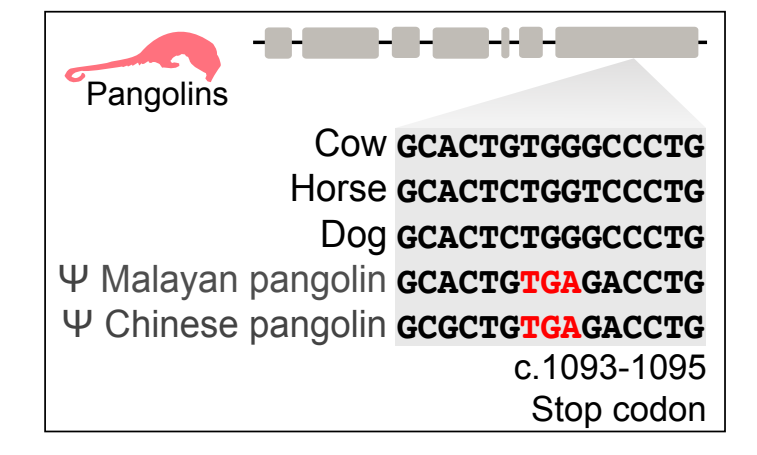

E
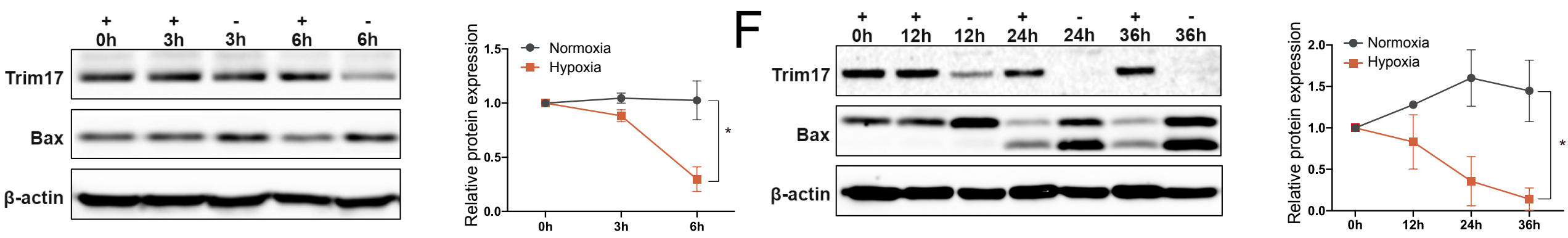

$\mathrm{G}$

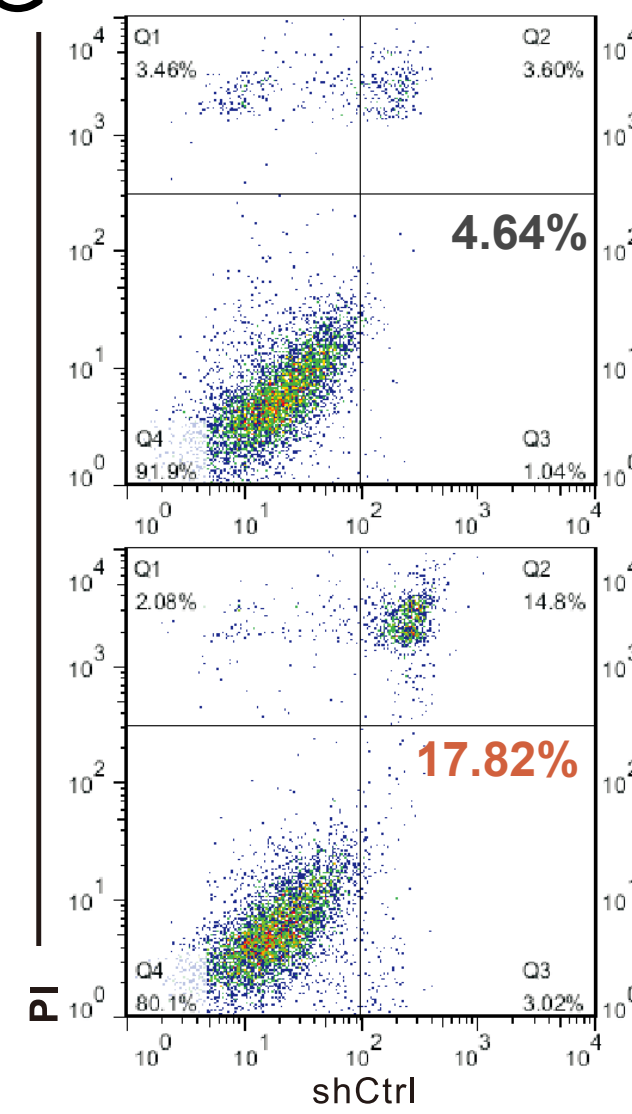

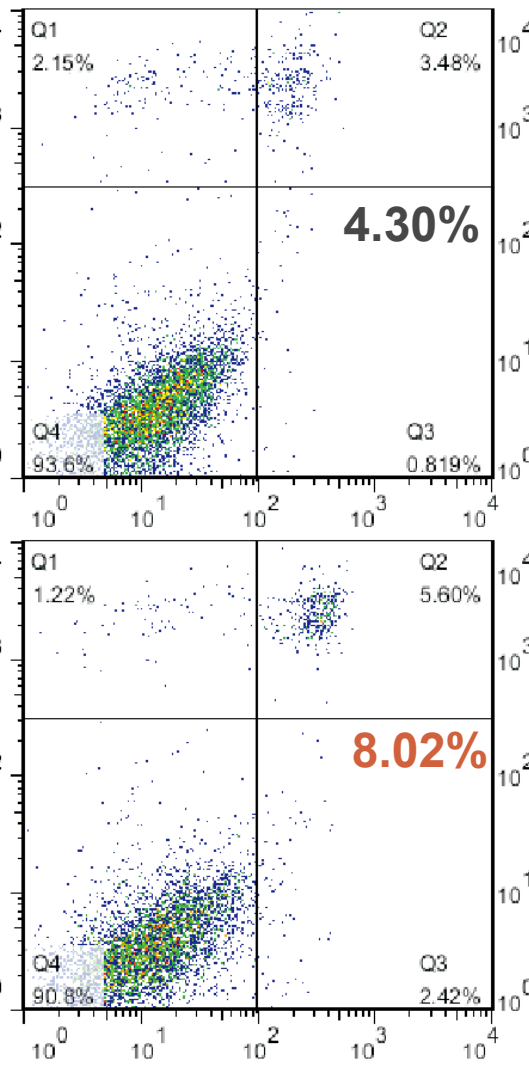

shTrim 17-1

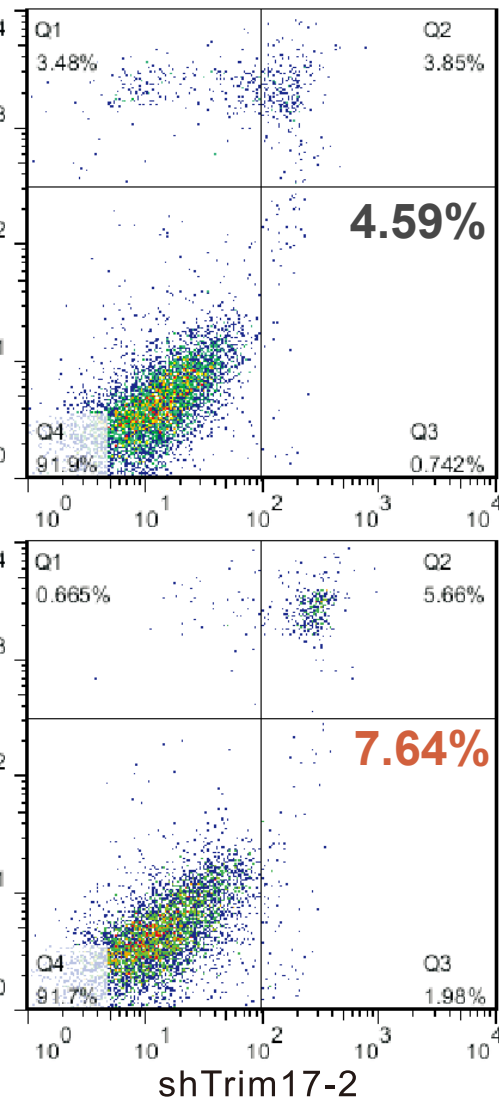

$\mathrm{H}$

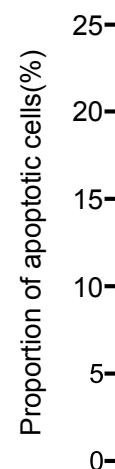

Hypoxia

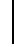

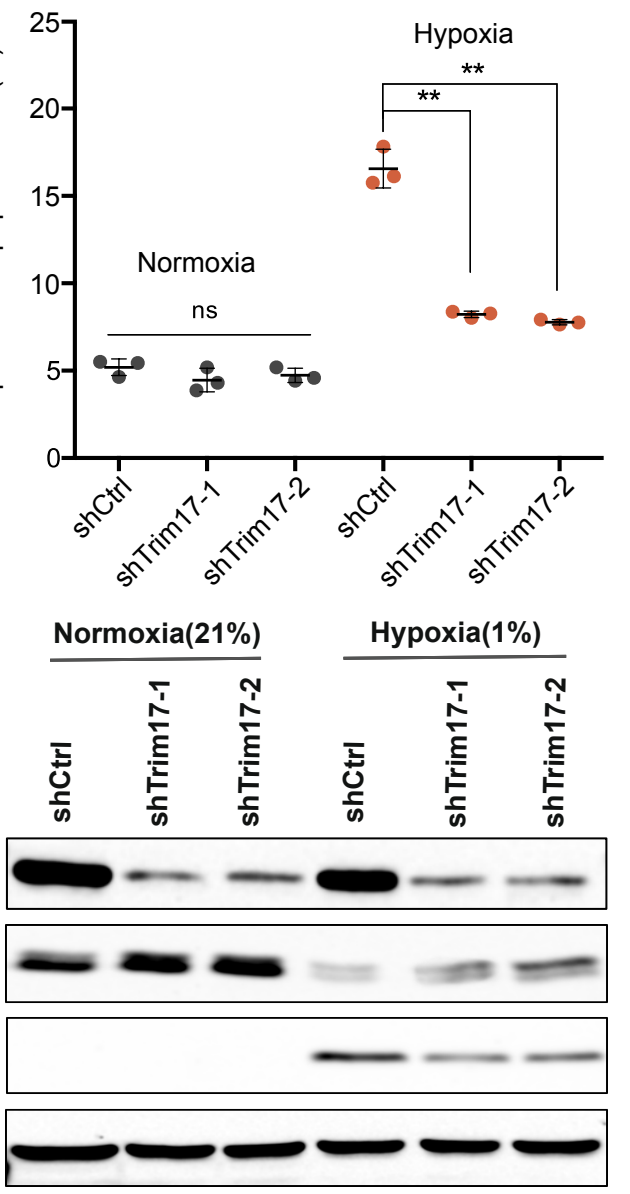

Annexin V-FITC 
A

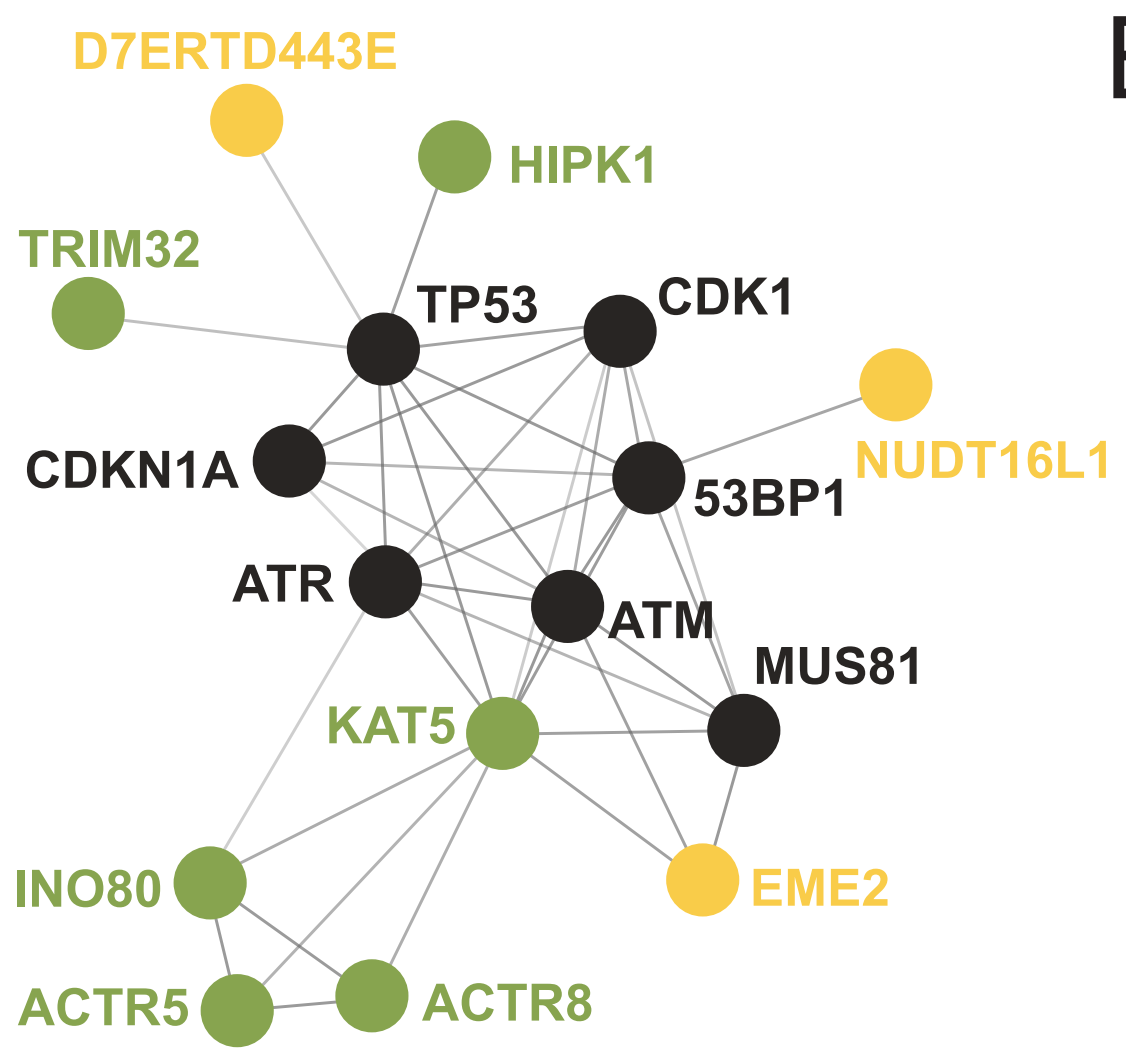

B
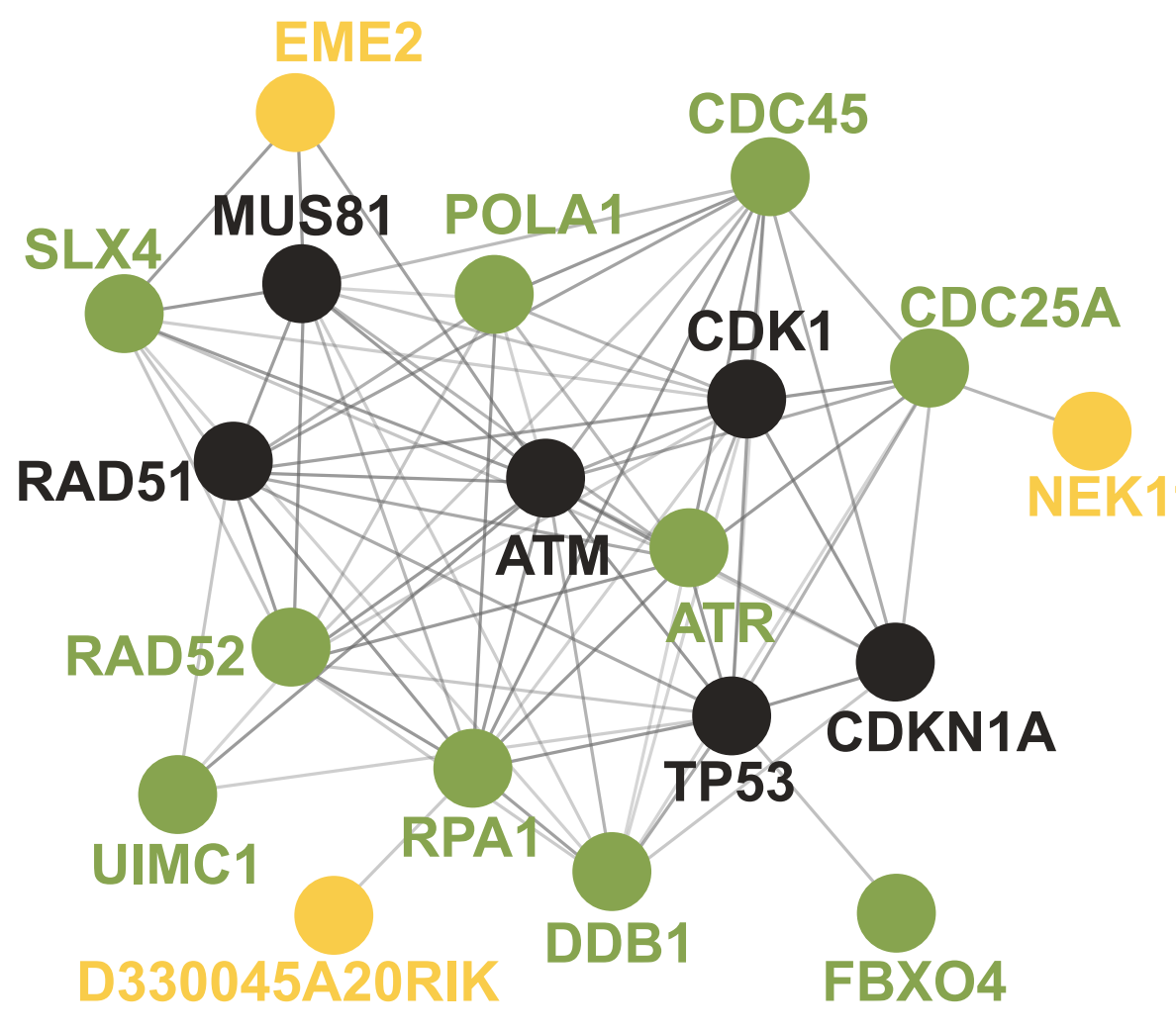
Mouse longest

protein sequnces

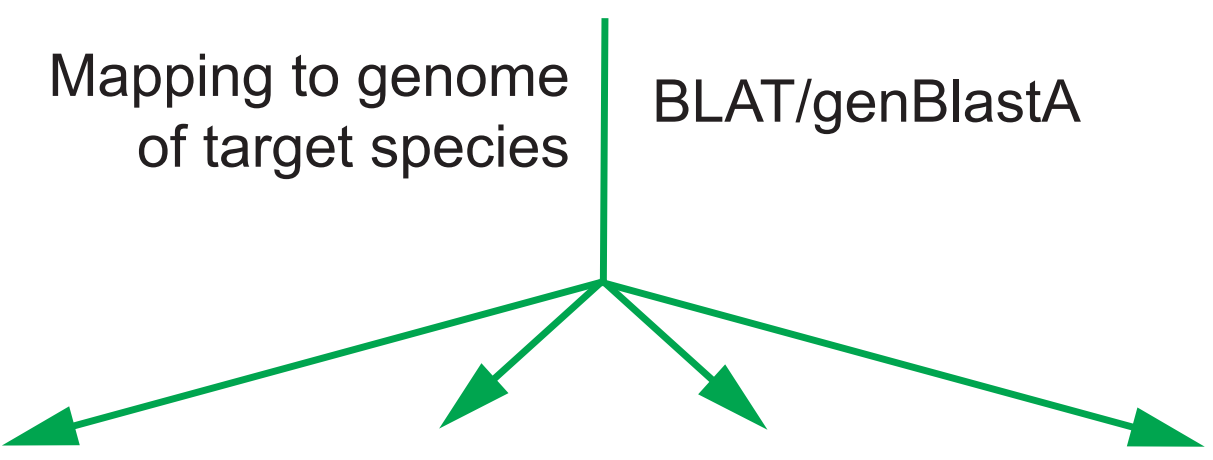

bioRxiv preprint doi: https:// doi.org/10.1101/2021.05.28.446201; this version posted May 29, 2021 The copyright holder for this preprint (which was not certified by peer review) is the authorfunder. All rights reserved. No rese sllowed without permission.

.

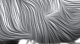

Naked mole rat

Blind mole rat

Guinea pig

Rat

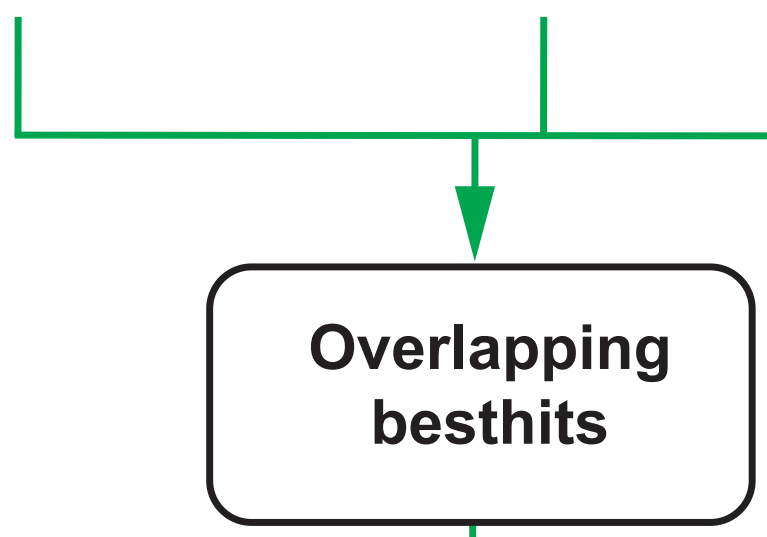

Detecting premature stop codons/frameshifts

GeneWise

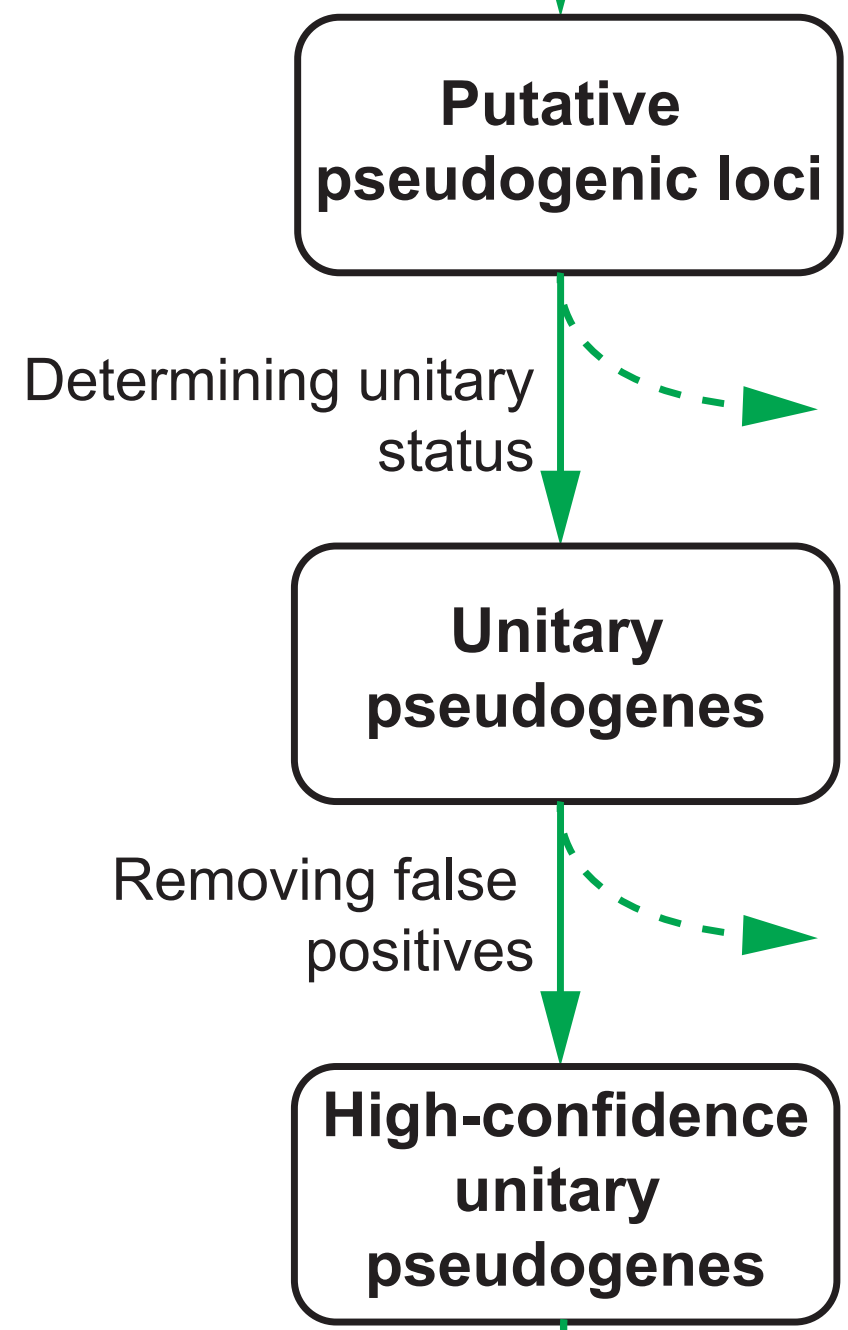

1,removing OR, ZNF and VR;

2,removing predicted proteins and intronless cDNA/expressed sequences

3 ,removing potential functional redundancy

4 ,removing genes not in a conserved genomic position

Detecting signature of relaxed selection (RELAX)
1 , removing errors by GeneWise

2 ,removing sequencing errors

3 ,removing mouse annotation errors

4,removing pseudogenes with only two compensatory frameshift mutations

Removing pseudogenes without a signature of relaxed selection

Gene functional loss events 